\title{
Design and optimization of ultra-wideband planar multilayer absorber based \\ on long-carbon fibers-loaded composites
}

\author{
Aicha El Assal $^{1}$, Hanadi Breiss ${ }^{1}$, Ratiba Benzerga ${ }^{1, *}$, Ala Sharaiha ${ }^{1}$ \\ ${ }^{1}$ CNRS, IETR - UMR 6164, Univ Rennes 1, F-35000 Rennes, France \\ *Corresponding Author: ratiba.benzerga@ univ-rennes1.fr
}

\begin{abstract}
This article presents a strategy for designing optimal microwave planar multilayer absorbers based on epoxy foam composites loaded with carbon fibers of $12 \mathrm{~mm}$ length. Firstly, the impedance gradient principle (gradual loaded composites) was adopted to realize two multilayer absorbers, of $125 \mathrm{~mm}$ thickness, using slightly loaded composites $(0.0125 \mathrm{wt}$ \% $<$ CFs $<0.075$ wt.\%) and relative highly loaded composites ( 0 wt. $\%<\mathrm{CFs}<0.4$ wt. $\%$ ), respectively. The simulation of these absorbers shows that composites with very low CF rates are sufficient to achieve a very close absorption performance and bandwidth to that of the commercial absorber, in the entire studied frequency range $(0.75-18 \mathrm{GHz})$. Secondly, the genetic algorithm optimizer is used to achieve a multilayer absorber that presents the best compromise between absorption performance and thickness. Different CF-loaded composites and layer thicknesses are therefore tested; a multilayer absorber with a total thickness of $98 \mathrm{~mm}$ is then proposed. This absorber shows a better reflection coefficient and a better compromise (absorption/total thickness) than that of the commercial absorber, while presenting a reduction of $22 \%$ in thickness. The presented simulation and measurement results confirm that a judicious choice of the composition and the thickness of each layer is necessary to optimize the absorption performance of a planar multilayer absorber. This paper also shows the advantage of using an optimizer to improve the absorption performance while reducing the total thickness of the absorber.
\end{abstract}


Keywords: Microwave absorption; Anechoic chamber; Multilayer absorber; Genetic algorithm; Carbon fiber based composites.

\section{Introduction}

Today, the rapid increase in telecommunication technologies induces the intensive use of devices operating in diverse areas, such as engineering, aviation, television and mobile communications. Therefore, many electronic devices and systems surrounding us produce an electromagnetic (EM) pollution, which ultimately creates a need for absorbing materials. These materials are therefore more and more employed not only for EM interference shielding [1-3], but also for other applications such as EM compatibility tests (aerospace and telecommunication domains) [4, 5], radar signature [6], medical systems [7, 8] and in EM characterization environments [9]. Researches around these materials have always concentrated on developing new lightweight and compact absorbing materials with a good absorption performance over a wide frequency band.

Absorbing materials are realized using different geometries and types of loads (dielectric, conductive and/or magnetic), depending on the targeted application. For example, magnetic tiles [10] and dielectric pyramids [10-13] or multilayer [10, 14, 15] absorbers were used for anechoic chamber application. In fact, the pyramidal and multilayer absorbers were used in these chambers to ensure a broadband absorption performance over a large bandwidth thanks to the impendence gradient which is ensured by these geometries [11]. This impendence gradient can be provided by a physical gradient, such as by pyramidal absorbers $[10,11,16]$, and in this case, the EM waves are dissipated in-depth of the pyramidal material. Moreover, the multi-reflections between the adjacent pyramids trap the EM waves by directing all these reflected waves toward the interior of the absorber where they will be absorbed [17]. Unlike pyramidal absorbers, multilayer absorbers, which are of our interest, must ensure a gradient of impedance by a 
gradient of the compositions (filler contents) of the different layers [14, 15, 18-21] and thus make the design of this type of absorbers with a broadband absorption performance more complex to realize. Thomassin et al. [15] have shown the importance of using the multilayer absorbers over the monolayer absorber. They have developed a multilayer absorber, in the Xband, based on carbon nanotubes (CNTs) dispersed in a polycaprolactone polymer foam with a gradual increase in the concentration of CNTs in depth. The multilayer absorber was composed of three layers with CNT concentrations of 0.5 wt. $\%, 1$ wt. $\%$ and 2 wt. $\%$. The proposed multilayer absorber shows a decrease in the reflection coefficient at least $5 \mathrm{~dB}$ (by approximately $70 \%$ ) and a broader absorption bandwidth compared to the monolayer of $1 \mathrm{wt} . \%$. Otherwise, the composition of each layer of the multilayer absorber should be chosen carefully in order to ensure a gradual impedance [22]. In this case, and in order to obtain a good absorption, the reflections must be minimized between air and absorber from the one hand, and at each interface (between the different layers) from the other hand. Indeed, if the incoming EM wave arrives at a medium with very different dielectric and/or magnetic properties, the medium will act as a reflector due to the impedance mismatching [20]. Thus, in the case of the multilayer absorber, if there is an impedance discontinuity between the layers, reflections will occur not only at the first interface, but also, between the different layers, with a reflection level proportional to the amount of the impedance discontinuity. For multilayer absorber, the first layer should have the closest dielectric and/or magnetic properties to air, and therefore the lowest possible complex permittivities and/or permeabilities, in order to ensure a smooth transition of EM waves at the first interface between the air and the absorber [16, 22]. This layer should then be followed by one or more layers, with intermediate complex permittivities and/or permeabilities which ensure the impedance continuity and a progressive absorption of the EM waves due to the intermediate losses [22]. The last layer(s) must have the highest losses in order to ensure a total absorption of the EM waves [16]. By this way, the EM waves will be transmitted from one layer to another with minimal reflections between the different interfaces, 
and with a gradual absorption inside the different layers. Therefore, the gradual dissipation is ensured when a gradual increase in the dielectric and/or magnetic properties of the layers composing the multilayer absorbers is assured [16]. The study of Zhang et al. [21] shows the importance of this gradual increase of the properties. Here, Ni nanoparticles were used as magnetic fillers in paraffin with $30 \mathrm{wt} . \%, 50 \mathrm{wt} . \%$ and $60 \mathrm{wt} . \%$ for a tri-layer multilayer absorber. Two cases were tested: a gradual increase of the wt.\% in depth for the first case, while a gradual decrease for the second case. As expected, the absorption performance of the first case, with a gradual increase from the lowest to the highest $\left(\varepsilon_{\mathrm{r}}\right.$ and $\left.\mu_{\mathrm{r}}\right)$, is more effective $(\Gamma<-10$ $\mathrm{dB})$ than the second case $(\Gamma>-10 \mathrm{~dB})$ in the whole frequency range [2-18] GHz.

However, it is difficult to control the impedance distribution, and to approach the ideal impedance (stepped impedance function) of the layers only by using the gradual loaded composite method $[16,23]$. The solution would be to calculate the module of the characteristic impedances of the different layers and to use them to build the multilayer absorber. For example, Micheli et al. have calculated the characteristic impedances of their composites based on epoxy resin loaded with different types of carbon and with different wt.\% in order to build a multilayer absorber [24]. However, when having a large panel of dielectric and/or magnetic composites, it is very difficult to choose among them the appropriate combination of the layer compositions. Furthermore, the choice of the layer thicknesses is also important in order to obtain the best absorption over a large bandwidth while keeping a compact absorber. Nevertheless, it is impossible to try all the possibilities (layer order, compositions and thicknesses); therefore, optimizers should be employed.

Genetic algorithm (GA) optimizer is one of the frequently used algorithms that was applied in different domains ranging from engineering [25, 26] to economics [27] and artificial intelligence [28] and its use becomes widespread in electromagnetics [29-31]. Among these applications, the GA was used for the conception of antennas [32-34] and antenna arrays [35, 36], of metamaterials [37-39], and for the design of microwave absorbers such as multilayer absorbers. 
For the latter, composites loaded with magnetic fillers were studied where both layers thicknesses and layers compositions [40-42] or only thicknesses [43] have been optimized using genetic algorithm. For example, Singh et al. [40], Padhy et al. [41] and Kumar et al. [42], have used the genetic algorithm for the optimization of planar absorber in $\mathrm{X}$ band frequency range, but, none of these studies proposed a multilayer absorber with a reflection coefficient less than $10 \mathrm{~dB}$ in the whole studied frequency range [8.2-12.4] GHz.

In this paper, the objective is to use the GA optimizer to achieve a planar dielectric multilayer absorber with the minimum thickness and that operates over a wide frequency band [0.75-18] GHz. Here, dielectric composites, based on epoxy foams loaded with different rates of carbon fibers (CFs) of $12 \mathrm{~mm}$ length will be used. In our previous work [44], we studied the effect of the $\mathrm{CF}$ length on the dielectric properties and we compared the dielectric properties $\left(\varepsilon_{r}^{\prime}\right.$ and $\tan \delta$ ) of the composites loaded with $0.25 \mathrm{wt} . \%$ of three different CF lengths ( $3 \mathrm{~mm}, 6 \mathrm{~mm}$ and $12 \mathrm{~mm}$ ). This comparison showed that, at low frequencies, the highest dielectric losses $\tan \delta$ are obtained by the composite loaded with $12 \mathrm{~mm}-\mathrm{CFs}(\tan \delta \mathrm{max}=1.12)$; this composite presents also low real permittivity value $\left(\varepsilon_{r(\max )}^{\prime}=3.7\right)$. One can note that the low frequencies, typically around $2 \mathrm{GHz}$, are the critical frequency range for the dielectric absorbers [44]. So, the combination between the low permittivity $\left(\varepsilon_{\mathrm{r}}^{\prime}\right)$ and the high dielectric losses $(\tan \delta)$, at low frequencies, candidates the composites loaded with CFs of $12 \mathrm{~mm}$ length to achieve a broadband multilayer absorber.

This paper is organized as follows; the elaboration and characterization techniques of the loaded epoxy foam composites are first presented. Then, the analytical calculation of the reflection coefficient of a monolayer and multilayer absorbers is presented, followed by the optimization principle using the genetic algorithm. After this, multilayer absorber prototypes achieved using, from the one hand, the gradual loaded composites and, from the other hand, the GA optimizer, will be conducted. The simulation and measurement results of the achieved 
prototypes will be presented and discussed; these results will be compared to that of the commercial absorber AH 125. Finally, the most relevant conclusions are laid out.

\section{Techniques and theory}

\subsection{Material elaboration method}

The epoxy resin (PB 170) and hardener (DM02), purchased from SICOMIN, and carbon fibers (fibers exPAN), provided by Apply Carbon SA, were used for the elaboration of the CFloaded epoxy foam composites. The used CFs have a density of 1.7 , a diameter of $7 \mu \mathrm{m}$ and a length of $12 \mathrm{~mm}$. Different weight percentages (wt.\%) from $0 \mathrm{wt} . \%$ to $0.4 \mathrm{wt} . \%$ were used. The different steps of the elaboration method are summarized in Fig. 1. The CFs (with the chosen wt.\%) were dispersed in the acetone $(60 \mathrm{ml})$ using the chemical surfactant SDS (sodium dodecyl sulfate) from Sigma-Aldrich and ultra-sonication method (using Sonics Materials VCX-750220). The mixture was then placed in the oven at $50^{\circ} \mathrm{C}$ for 72 hours to evaporate the solvent (acetone). After this, the epoxy resin was added to this mixture, as well as the hardener with the proportions Resin/Hardener of 10:3.6. The mixture was then placed into a mold for foaming and polymerization steps during 6 hours at room temperature. Subsequently, the mold was placed in the oven (at $60^{\circ} \mathrm{C}$ for 6 hours) to finalize the polymerization of the epoxy foam thus obtaining the final rigid composite. Finally, the samples were cut to the dimensions of $15 \mathrm{~cm}$ x $15 \mathrm{~cm}$ x 6 $\mathrm{cm}$, needed for the characterization in the anechoic chamber. The measured density of all the elaborated composites is $0.13 \mathrm{~g} \cdot \mathrm{cm}^{-3}$. 


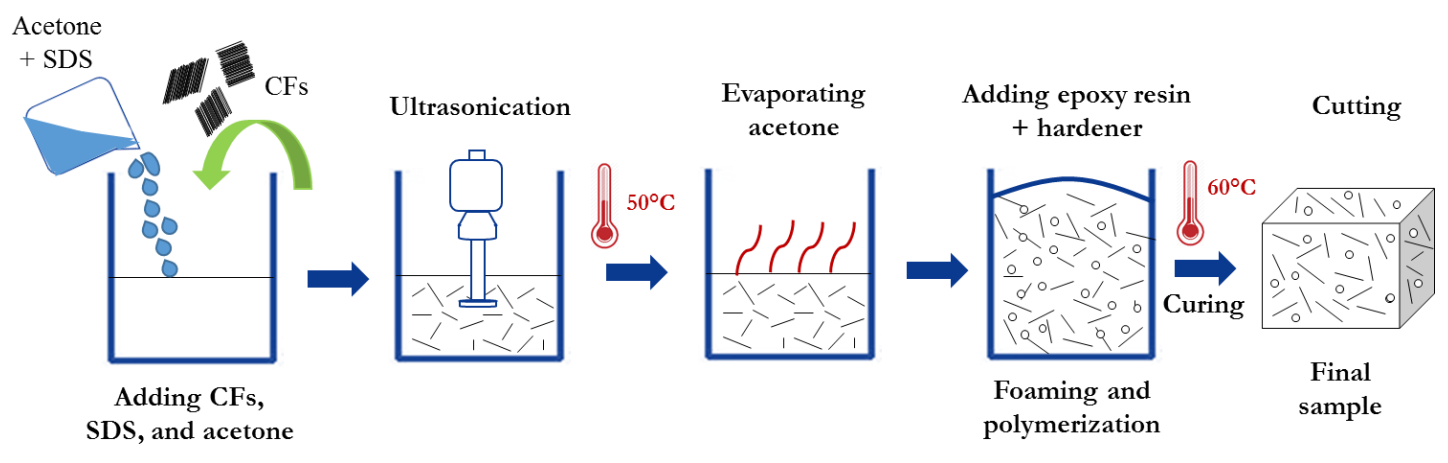

Fig. 1 Elaboration steps of the CF loaded epoxy foam composites

\subsection{Characterization technique}

The complex permittivity of the elaborated composites is extracted from the measurement of the reflection $\left(S_{11} \& S_{22}\right)$ and transmission $\left(S_{21} \& S_{12}\right)$ coefficients using free space method, detailed in [45], where a quasi-monostatic configuration is used. For this latter, two horn antennas (3115 Ets-Lindgren Model), that operate in the frequency range $0.75-18 \mathrm{GHz}$, are placed side by side in the anechoic chamber and are connected to a vector network analyzer. Sample is placed in front of the two antennas at a distance d, ensuring the far field condition (Eq. (1)), where $\mathrm{D}$ is the antenna aperture diameter and $\lambda$ is the highest measured wavelength (at the lowest frequency) [45]:

$$
\mathrm{d} \geq \frac{2 \mathrm{D}^{2}}{\lambda}
$$

The measured coefficients are then used to extract the real and imaginary parts of the dielectric permittivity $\left(\varepsilon_{\mathrm{r}}^{\prime}\right.$ and $\left.\varepsilon_{\mathrm{r}}^{\prime \prime}\right)$ of the composite under test using Fenner et al. formulas [45, 46]. The dielectric losses of these composites, which indicate the capacity of dissipation of the energy inside the composite material, are then calculated by $\tan \delta=\frac{\varepsilon_{\mathrm{r}}^{\prime \prime}}{\varepsilon_{\mathrm{r}}^{\prime}}$.

The same configuration is used for the measurement of the reflection coefficient of the achieved prototypes. For a normal incidence, the two antennas are placed side by side with a small negligible angle of incidence as shown in Supplementary Data 1 (a). For an oblique 
incidence $\theta$, the two antennas are separated by the needed distance in order to form the required angle of incidence; Supplementary Data 1 (b) shows the configuration of the two antennas with an incidence angle of $30^{\circ}$.

\subsection{Multilayer absorber design and optimization}

\subsubsection{Mathematical model}

According to the transmission line theory $[47,48]$, for a single-layer microwave absorber with a thickness $\mathrm{d}$, the total reflection coefficient at a given frequency $\mathrm{f}$ can be calculated using the complex relative permeability $\left(\mu_{\mathrm{r}}\right)$ and the complex relative permittivity $\left(\varepsilon_{\mathrm{r}}\right)$ of the monolayer and using Eqs. (2) and (3) where Zin is the entrance impedance to the single-layer absorber, $\mathrm{Z}_{0}$ is the impedance of air, and $\mathrm{c}$ is the velocity of light $\left(3 * 10^{8} \mathrm{~m} / \mathrm{s}\right)$.

$$
\Gamma(d B)=20 \log \left|\frac{Z_{\text {in }}-Z_{0}}{Z_{\text {in }}+Z_{0}}\right|
$$

With

$$
Z_{\text {in }}=Z_{0} \sqrt{\frac{\mu_{r}}{\varepsilon_{r}}} \tanh \left[j\left(\frac{2 \pi f d}{c}\right) \sqrt{\frac{\mu_{r}}{\varepsilon_{r}}}\right]
$$

For a multilayer microwave absorber (Fig. 2), the computation of the total reflection coefficient takes in account the multiple reflections that occur at each interface. Here, and according to Balanis [49], the reflection coefficient $\Gamma$ is computed by adding all the single reflections $\left(\Gamma_{0}, \Gamma_{1}, \ldots, \Gamma_{\mathrm{N}}\right)$ at each interface, multiplying each one by its phase as shown in Eq. (4) where $\beta_{N}$ is the propagation constant of the Nth layer (calculated using Eq. (5)) and $\Gamma_{0}$, $\Gamma_{1}, \ldots, \Gamma_{\mathrm{N}}$ are calculated using Eq. (6) and the characteristic impedance of the layers using Eq. (7) with $\mathrm{N}=0,1,2,3 \ldots$

$$
\begin{gathered}
\Gamma=\Gamma_{0}+\Gamma_{1} e^{-j 2 \beta_{1} d_{1}}+\Gamma_{2} e^{-j 2\left(\beta_{1} d_{1}+\beta_{2} d_{2}\right)}+\cdots+\Gamma_{N} e^{-j 2\left(\beta_{1} d_{1}+\beta_{2} d_{2}+\cdots+\beta_{N} d_{N}\right)} \\
\beta_{N}=\frac{2 \pi f}{c} \sqrt{\varepsilon_{r N} \mu_{r N}}
\end{gathered}
$$




$$
\begin{gathered}
\Gamma_{N}=\frac{\eta_{N+1}-\eta_{N}}{\eta_{N+1}+\eta_{N}} \\
\eta_{N}=Z_{0} \sqrt{\frac{\mu_{r N}}{\varepsilon_{r N}}}=Z_{0} \sqrt{\frac{\mu_{r N}^{\prime}-j \mu_{r N}^{\prime \prime}}{\varepsilon_{r N}^{\prime}-j \varepsilon_{r N}^{\prime \prime}}}
\end{gathered}
$$

Here, the multiple secondary reflections at each boundary of the multilayer absorber are neglected. This approximation is used because these multiple secondary reflections are very small since the layers composing the absorber consist of absorbing layers [49]. Using this approximation and the different equations from Eq. (4) to Eq. (6), a MATLAB function that computes the total reflection coefficient of multilayer absorber was developed. This code allows the calculation of the reflection coefficient using the complex dielectric permittivities and/or magnetic permeabilities of the layers composing the multilayer absorber. In our case, the elaborated composites are pure dielectric materials $\left(\mu_{\mathrm{r}}=1\right)$, so only complex dielectric permittivities are taken into consideration.

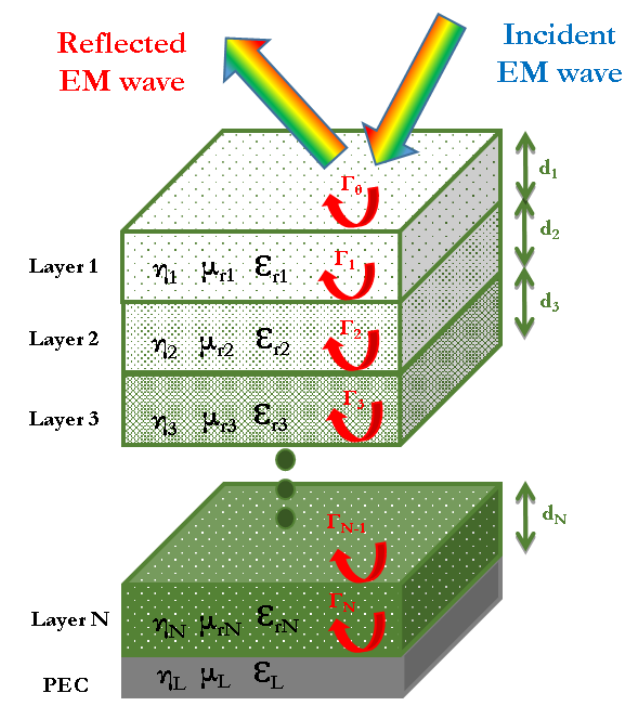

Fig. 2 Reflection coefficients of the multilayer absorber

\subsubsection{Optimization using genetic algorithm}

The analytical calculation of the reflection coefficient is then combined to GA optimizer. The benefit of using such optimization is to compute the reflection coefficient (which is the goal to 
be minimized) for several possible combinations (composition and thickness of each layer) and ends up with the best combination that gives the lowest requested reflection coefficient.

The function that ensures the connection between the physical problem (calculation of the reflection coefficient of the multilayer absorber at normal incidence) and the GA optimizer is the fitness function [50]. It relates the function that computes the reflection coefficient to the variable parameters (compositions and thicknesses of each layer) to be changed by the GA optimizer. At each iteration, the fitness function compares the computed $\left(\Gamma_{\text {computed }}\right)$ to the targeted $\left(\Gamma_{\text {Target }}\right)$ reflection coefficients; this operation is repeated until the goal of the best absorption performance (lowest reflection coefficient) is reached. Here, the GA will stop in two cases: when the number of generations exceeds the imposed maximum limit of iterations or when the GA achieves its goal $\left(\Gamma_{\text {Target }}\right)$. The introduced parameters for the optimization program are the number of layers in the multilayer absorber, the thickness limits (min and max) of each layer, the frequency range, the material database and the targeted reflection coefficient (Supplementary Data 2). The optimization by the developed code takes only a few minutes to be finished. After this, the reflection coefficient of the proposed design is computed using CST for the oblique incidence of $30^{\circ}$ as the developed program deals only with the normal incidence. One can note that a verification of the reflection coefficient, calculated by the optimizing program, was made by 3D Electromagnetic Simulation Software (CST) for normal incidence; an example of the comparison between the calculated and simulated reflection coefficients will be presented further in this article.

\section{Results and discussion}

\subsection{Dielectric properties of CFs loaded epoxy foam composites}

The dielectric properties $\left(\varepsilon_{\mathrm{r}}^{\prime}\right.$ and $\left.\tan \delta\right)$ of the epoxy foam composites loaded with different rates (between 0 wt.\% and 0.4 wt.\%) of CFs of $12 \mathrm{~mm}$ length are shown in Fig. 3. The latter shows, as expected, that both $\varepsilon_{\mathrm{r}}^{\prime}$ and tan $\delta$ increase with the increase of the weight percentage of 
the CF load, thus giving a large panel of dielectric properties. For example, @ $4 \mathrm{GHz}$, $\varepsilon_{\mathrm{r}}^{\prime}$ increases from $\varepsilon_{\mathrm{r}}^{\prime}=1.3$ (with 0.0125 wt.\%) to $\varepsilon_{\mathrm{r}}^{\prime}=3.3$ (with 0.4 wt.\%) while tan $\delta$ increases from $\tan \delta=0.15$ (with 0.0125 wt. $\%$ ) to $\tan \delta=1.32$ (with 0.4 wt.\%). In addition to that, very close values, especially for the real part of permittivity, are obtained with composites loaded with very low weight percentages $(\leq 0.025$ wt.\%). For example, @ $4 \mathrm{GHz}$, the real permittivity values $\varepsilon_{\mathrm{r}}^{\prime}$ are equal to $1.3,1.3$ and 1.32 for 0.0125 wt. $\%, 0.01875$ wt. $\%$ and 0.025 wt.\%, associated with dielectric losses of $0.15,0.21$ and 0.34 , respectively. One can note that after 10 $\mathrm{GHz}$, very close values of $\varepsilon_{\mathrm{r}}^{\prime}$ for all composites are obtained; for example, @ $12 \mathrm{GHz}, \varepsilon_{\mathrm{r}}^{\prime}=1.13$ (with 0.025 wt.\%), $\varepsilon_{\mathrm{r}}^{\prime}=1.37$ (with 0.1 wt.\%) and $\varepsilon_{\mathrm{r}}^{\prime}=1.5$ (with 0.4 wt.\%).

Fig. 3 also shows that, except the unloaded epoxy foam which presents constant dielectric properties $\left(\varepsilon_{\mathrm{r}}^{\prime}=1.2\right.$ and $\left.\tan \delta=0.0009\right)$ over the studied frequency band $[0.75-18] \mathrm{GHz}$, a standard decreasing behavior of the real permittivity in function of frequency is obtained by all composites and it is accompanied by an increase to a maximum followed by a decrease of the dielectric losses, especially for composites loaded with low weight percentages $(<0.2 \mathrm{wt} . \%)$. This behavior is often observed for composites loaded with low weight percentages of long CFs which show a maximum of their dielectric losses at low frequencies, typically below $5 \mathrm{GHz}$, as seen in [44]. Among our elaborated composites, the permittivity of the sample loaded with 0.1 wt.\%, for example, decreases from 3.38 (@ $0.75 \mathrm{GHz}$ ) to 1.2 (@ $18 \mathrm{GHz}$ ) associated with an increase in the losses from $\tan \delta=0.62$ (@0.75 GHz) to $\tan \delta_{(\max )}=0.81$ (@ $\left.4.8 \mathrm{GHz}\right)$ followed by a decrease of this latter to $\tan \delta=0.47$ (@18 GHz).

Furthermore, low values of real permittivity associated with high dielectric losses could be noted from Fig. 3. Here, the highest value of the dielectric properties is obtained with the epoxy foam loaded with 0.4 wt. $\%$ where $\varepsilon_{\mathrm{r}(\max )}^{\prime}=5 @ 0.75 \mathrm{GHz}$ associated with high $\tan \delta$ of 1.24 . Indeed, when very low weight percentages $(<0.1 \mathrm{wt} . \%)$ are used, permittivity values are very low and they range from 1.5 to $2.5 @ 0.75 \mathrm{GHz}$ and change very slightly for high frequencies; 
these permittivity values are associated with high values of $\operatorname{losses}\left(\tan \delta_{(\max )}\right.$ of 0.15 and 0.64 with 0.0125 wt. $\%$ and 0.075 wt. $\%$, respectively).

Since these composites present low $\varepsilon_{\mathrm{r}}^{\prime}$ and high tan $\delta$, especially at the low frequencies which are critical for the absorption application, a good absorption performance can be expected with these composites.

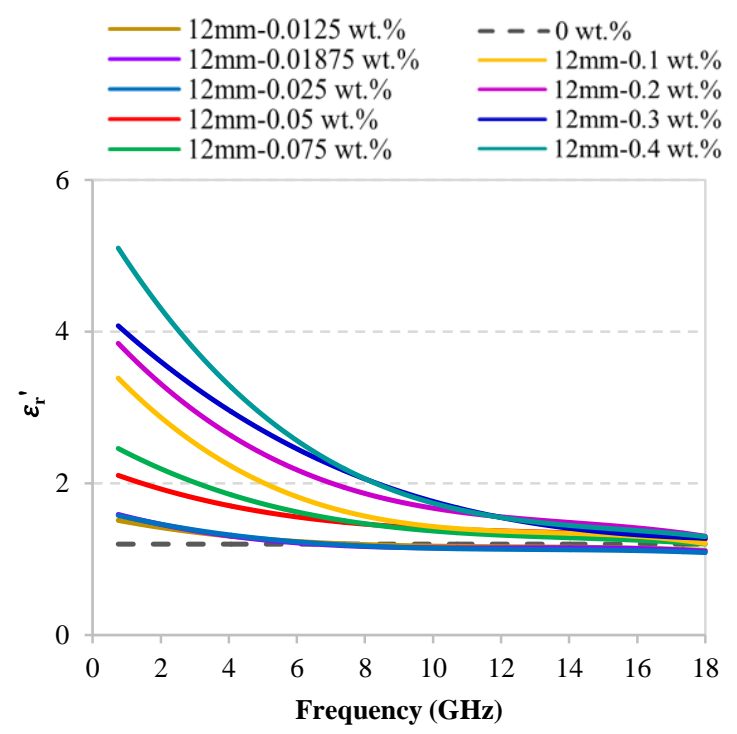

(a)

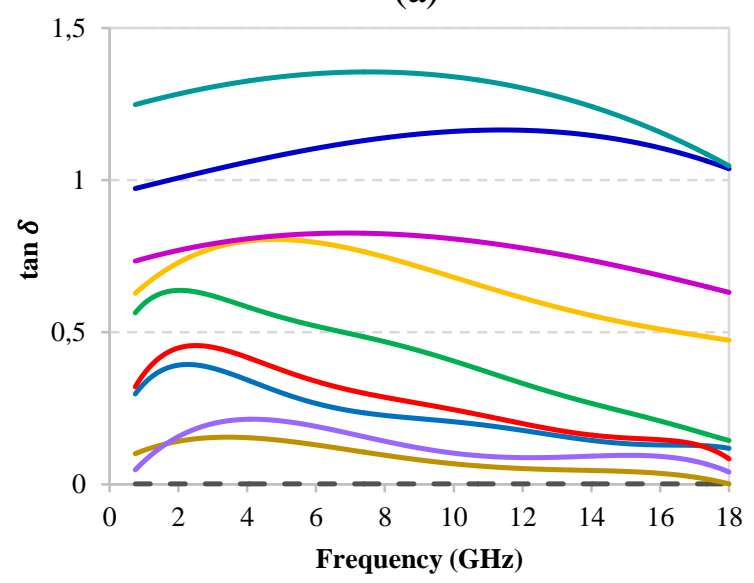

(b)

Fig. 3 Dielectric properties (a) $\varepsilon_{r}^{\prime}$ and (b) tan $\delta$ of the composites loaded with $12 \mathrm{~mm}$ CFs using different wt.\%

\subsection{Multilayer absorber design by using the gradient of composition principle}

Our goal was to use the elaborated composites to achieve a broadband multilayer absorber. Our composites can be divided into two categories: very slightly loaded (between $0 \mathrm{wt} . \%$ and 
0.1 wt. $\%$ ) and relative highly loaded (between 0.1 wt.\% and 0.4 wt. $\%$ ) composites. Two gradients of compositions can thus be obtained and tested in order to observe the influence of the load rates, and thus the dielectric properties, on the absorption performance and bandwidth of the planar absorbers.

Two multilayer absorber designs, with five layers for each one, are then proposed. The first multilayer absorber (MLA125-1) is designed with very low CF-loaded composites (from 0.0125 wt.\% to 0.075 wt.\% with a step of 0.0125 wt.\%) and the second design (MLA125-2) is composed of composites loaded with relative high CF loads ( 0 wt.\% to 0.4 wt. $\%$ with a step of 0.1 wt.\%). Multilayer absorbers are constructed by stacking the elaborated composites, increasing the load (wt.\%) gradually in depth and with a total thickness of $125 \mathrm{~mm}$ (thickness of each layer is equal to $25 \mathrm{~mm}$ ), as shown in Fig. 4 (a) and (b), respectively, for MLA125-1 and MLA125-2. These absorbers present the same dimensions as the commercially available PU foam based multilayer absorber $\mathrm{AH} 125$ that is used in the anechoic chamber for the same frequency band [0.75-18] GHz [10].

Using CST Microwave Studio, the reflection coefficients $(\Gamma(\mathrm{dB}))$ of the proposed multilayer absorbers (MLA125-1 and MLA125-2), backed with a perfect electric conductor (PEC), are computed and the results are shown in Fig. 4 (c) and (d). For a normal incidence of the EM waves (Fig. 4 (c)), a reflection coefficient less than $-10 \mathrm{~dB}$ is expected in the frequency ranges of [1.13-18] GHz and [0.97-18] GHz for the MLA125-1 and MLA125-2, respectively. A reflection coefficient less than $-20 \mathrm{~dB}$ is obtained for frequencies higher than $4 \mathrm{GHz}$ for the MLA125-1 while the reflection is higher than $-20 \mathrm{~dB}$ in practically all the studied frequency band [0.75-17] GHz for the MLA125-2. For oblique incidence of $30^{\circ}$ (TM mode in Fig. 4 (d) and TE mode in Supplementary Data 3), the same observations can be done. Indeed, MLA125-1 shows better absorption performance (lower reflection coefficients) than the second absorber design (MLA125-2). Here, a reflection coefficient less than $-10 \mathrm{~dB}$ is obtained from $1.18 \mathrm{GHz}$ for both TE and TM polarizations by MLA125-1 and from $6 \mathrm{GHz}$ (for TE) and from $0.85 \mathrm{GHz}$ (for TM) 
by MLA125-2. Moreover, a reflection coefficient less than $-20 \mathrm{~dB}$ is obtained from $2.35 \mathrm{GHz}$ by MLA125-1 (and from 4.6 GHz for TM and TE modes, respectively), and could not be obtained by MLA125-2.

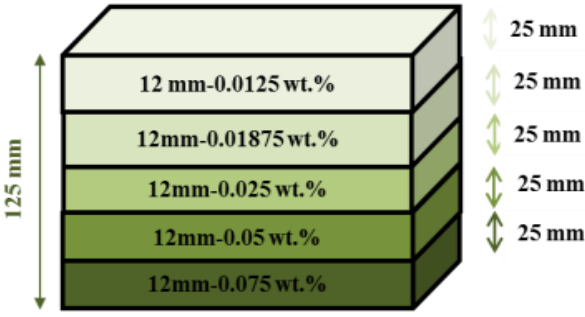

(a)

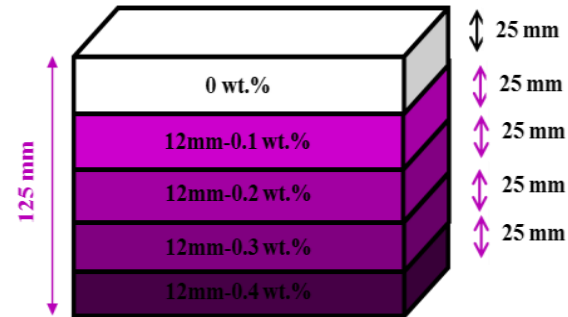

(b)

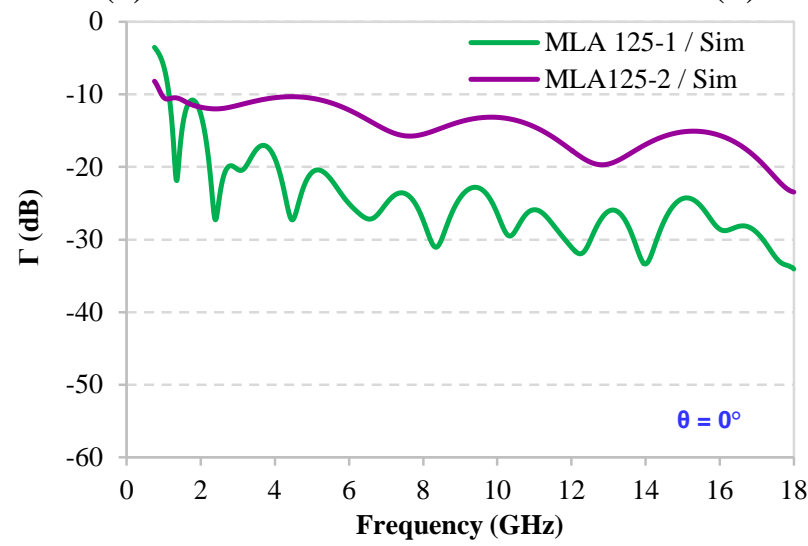

(c)

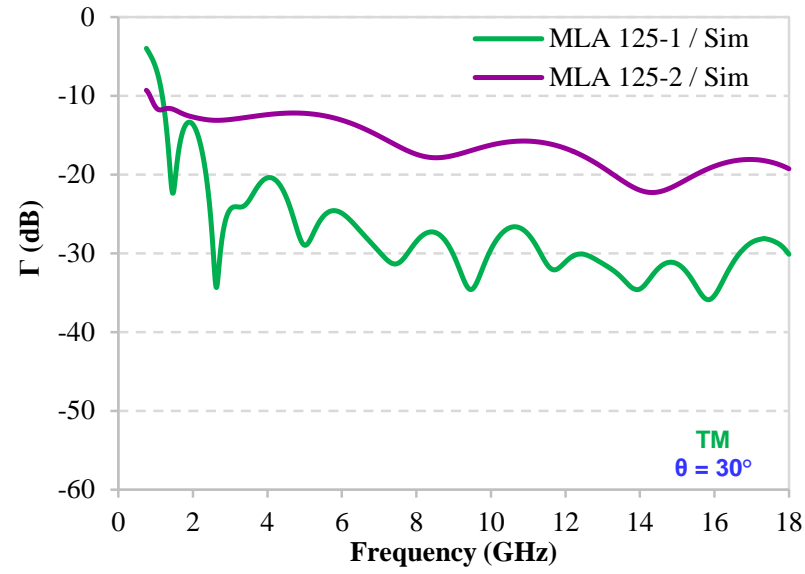

(d)

Fig. 4 Designs of MLA125-1(a) MLA125-2 (b) and simulated reflection coefficients of these multilayer absorbers for normal (c) and oblique incidence $\theta=30^{\circ}$ for $\mathrm{TM}$ polarization (d)

From the presented results, it was seen that using relative high loads for the gradual compositions of the layers of MLA125-2, a broadband absorption bandwidth with $90 \%$ of absorption is ensured between 0.9 and $18 \mathrm{GHz}$, but using the slightly loaded composites for the 
MLA125-1, a better absorption performance than that of MLA125-2 with a practical absorption higher than $99 \%$ is obtained between 2.3 and $18 \mathrm{GHz}$. This is mainly due to the better impedance transition realized by the MLA125-1, unlike that of the MLA125-2. Supplementary Data 4 groups the calculated module of the characteristic impedances (and the used formula for this calculation) for the different layers of the two absorber designs. This Fig. shows, gradual and very close impedance values between the different layers for the MLA125-1 design (Supplementary Data 4 (a)) and also between all the layers composing MLA125-2, except between the first and the second layer where a very large gap is observed (Supplementary Data 4 (b)). Indeed, even if gradual $\mathrm{CF}$ rates are used for both multilayer absorbers, a very big difference is observed in the characteristic impedances of the first two layers of this design.

In order to confirm that this gap is at the origin of the observed poor performance of the MLA125-2, the reflected and transmitted powers of the EM waves at each interface are calculated for the two absorber designs. For this, the reflection and transmission coefficients are calculated using Eqs. (6) and (8), respectively, and using the characteristic impedances of each two adjacent layers $\left(\eta_{\mathrm{N}}\right.$ and $\left.\eta_{\mathrm{N}+1}\right) ; \eta_{0}=\mathrm{Z}_{0}=377$ is the impedance of air, $\mathrm{N}=1,2,3 \ldots$ and $\mu_{\mathrm{rN}}=$ 1, since our composites are pure dielectric. For more details about the used annotations, see Supplementary Data 5 (a).

$$
T_{N}=\frac{2 \eta_{(N+1)}}{\eta_{(N+1)}+\eta_{N}}
$$

After that, and to calculate the transmitted power density of the EM waves to the next layer $\mathrm{P}_{\mathrm{N}}$, Eq. (9) is used where the attenuation $\alpha \mathrm{N}$ in each layer is calculated using Eq. (10), with $\mathrm{P}_{1}=$ 1 and $\mathrm{N}=2,3,4 \ldots$

$$
\begin{gathered}
P_{N}=P_{N-1} e^{-2 \alpha_{N} d_{N}} \\
\alpha_{N}^{2}=\frac{\omega^{2} \varepsilon_{r}^{\prime}}{2 c^{2}}\left(\sqrt{1+(\tan \delta)^{2}}-1\right)
\end{gathered}
$$


Finally, using Eq. (11) and Eq. (12) and $\mathrm{N}=1,2,3 \ldots$, the reflected and transmitted power densities (in \%) of the incoming power $\left(\mathrm{P}_{\mathrm{N}}\right)$ at each interface are calculated and are presented in Fig. 5 (a) and (b) for MLA125-1 and MLA125-2, respectively.

$$
\begin{gathered}
P_{r N}=100 *\left(P_{N} *\left|\Gamma_{N}\right|^{2}\right) \\
P_{t N}=100 *\left(P_{N} *\left|T_{N}\right|^{2} * \operatorname{real}\left(\frac{\eta_{N}}{\eta_{N+1}}\right)\right.
\end{gathered}
$$

For the slightly loaded MLA125-1 (Fig. 5 (a)), the percentages of power reflections (Pr1 to Pr3) are very low at the different interfaces, meaning that there is practically no reflection of the transmitted power densities at these interfaces. The highest reflected power is $1.37 \% @ 1 \mathrm{GHz}$, and it is obtained between the third and the fourth layers (reflected power Pr4). Contrary to that, for the highly loaded MLA125-2 (Fig. 5 (b)), the reflected power at the second interface (between the first and the second layers Pr2) is very high (up to $10.5 \%$ @ $0.75 \mathrm{GHz}$ ). This result confirms that the poor absorption performance of this design is mainly due to the impedance mismatch that exists between the first and the second layers.

Otherwise, the percentage of the transmitted EM waves' power PtN from the incident powers (transmitted after absorption in the previous layer) of the MLA125-1 and MAL125-2 are presented in Supplementary Data 5 (b) and (c). These figures show that for the MLA125-1, there is an attenuation (absorption) of the transmitted EM waves in depth from one layer to another (Supplementary Data 5 (b)). Indeed, for this design, the reflections are very low at the interfaces and the transmissions become increasingly weaker in depth (Supplementary Data 5 (b)). This means that the power of the EM waves is absorbed gradually in the layers, and this starts from the first layer of the absorber as approved by the transmission at the level of the second interface (Pt2) that is less than $80 \%$ over the entire frequency range $2.1 \mathrm{GHz}<\mathrm{f}<17 \mathrm{GHz}$; this transmittance decreases to $63 \%$ at the frequency of $6.1 \mathrm{GHz}$. In contrast to this, MLA125-2 shows very low absorption at the first layer because more than $90 \%(\mathrm{Pt} 2)$ of the wave power is transmitted to the second layer in the entire frequency range (Supplementary Data 5 (c)). This is 
quite consistent because the first layer of this absorber is based on the unloaded epoxy foam, which presents very low losses $(\tan \delta=0.0009)$. Then, the second layer of this design is a highly absorbing layer; this has affected the percentage of the transmitted power to the third layer (Pt3), which becomes very low (less than $10 \%$ for frequencies $>4 \mathrm{GHz}$ ) (Supplementary Data 5 (c)). To summarize this part, the absorption performance of the MLA125-2 is mainly due to the reflection at the interface between the first and the second layers, and also to the strong absorption power of the second layer which is made of an epoxy foam loaded with $0.1 \mathrm{wt} . \%$ CFs, presenting a high dielectric loss (Fig. 3).

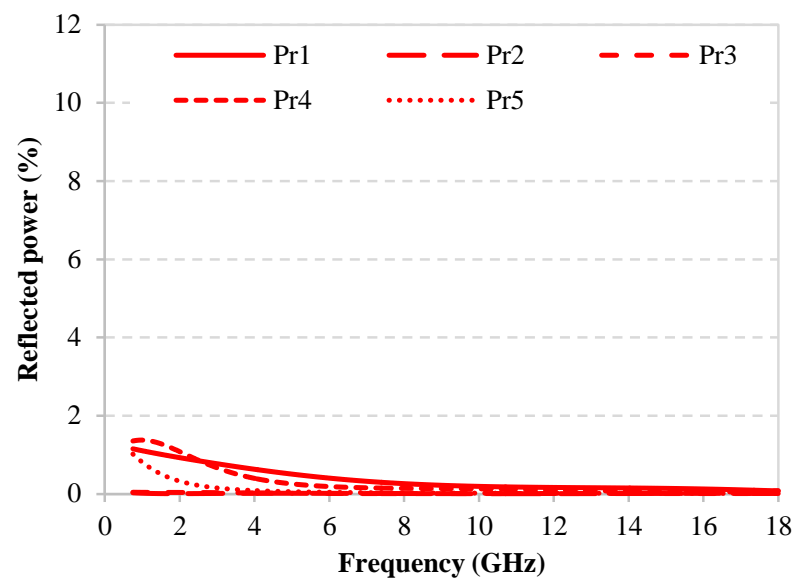

(a)

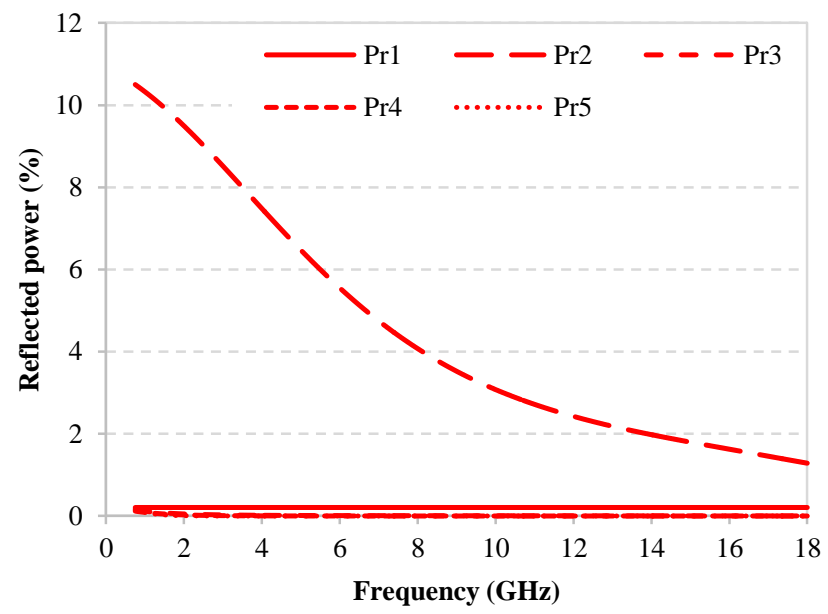

(b)

Fig. 5 Reflected $\left(\operatorname{Pr}_{N}\right)$ power densities for (a) MLA125-1 and (b) MLA125-2

As a conclusion, very small reflected power densities and high transmitted power densities between the layers and a good progressive absorption in depth should be ensured to obtain the best possible absorption performance, as seen by MLA125-1. 
As the MLA125-1 presents the best result in the entire studied frequency band, this design was realized and measured in order to verify the simulation results of this absorber. Then, this prototype was used as a reference for the rest of the article. The different layers composing the proposed multilayer absorber were cut to the desired thickness ( $25 \mathrm{~mm}$ for each composite) and glued together, using epoxy glue; the photograph of the realized prototype is shown in Fig. 6 (a). This prototype was measured in the anechoic chamber following the procedure explained in part 2.2. A comparison between the simulated and the measured reflection coefficients of the MLA125-1, for normal incidence, is presented in Fig. 6 (b). The obtained results confirm the high absorption performance, predicted by simulation, for this prototype. Indeed, a reflection coefficient less than $-10 \mathrm{~dB}$ from $1.3 \mathrm{GHz}$ and less than $-20 \mathrm{~dB}$ from $2.43 \mathrm{GHz}$ is obtained by the measurement (Fig. 6 (b)).

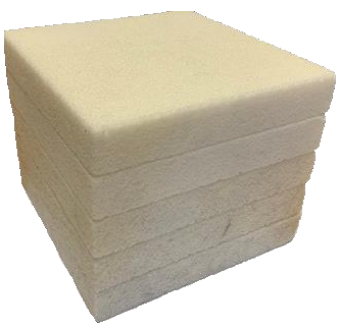

(a)

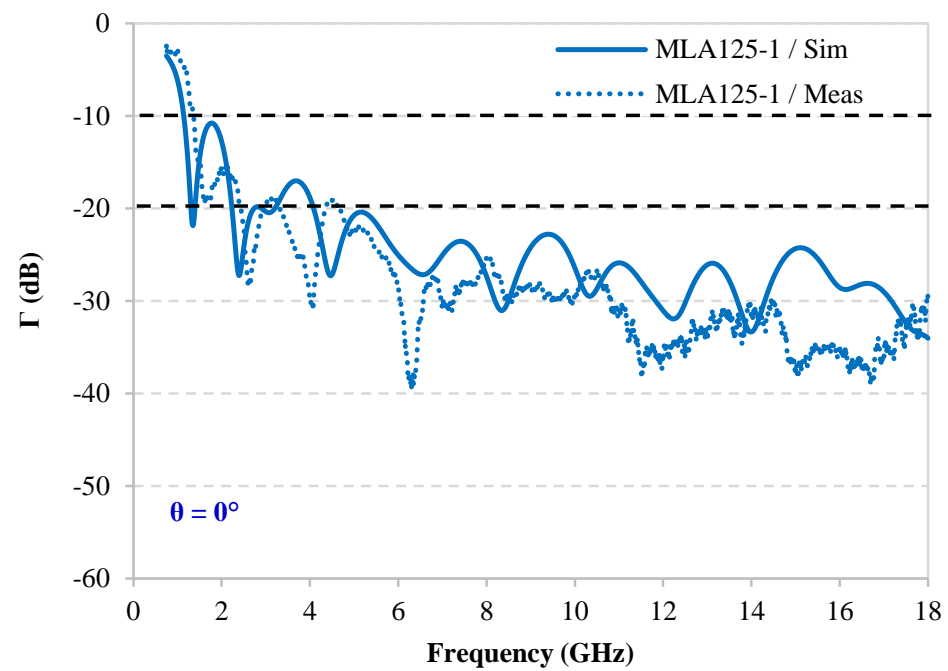

(b)

Fig. 6 (a) MLA125-1 achieved prototype and (b) its simulated and measured reflection coefficients for normal incidence 
For the oblique incidence of $30^{\circ}$ (TE and TM polarizations shown in Fig. 7 (a) and (b) respectively), the good absorption performance is also confirmed. Here, a slight difference with better measured reflection coefficients, compared to that of simulation, is obtained at high frequencies; this is more notable for TE polarization (Fig. 7 (b)) with, at least, an improvement of $4 \mathrm{~dB}$.

The obtained result for MLA125-1, at normal incidence, is compared to the measured reflection coefficient of the commercial multilayer absorber AH 125 (from SIEPEL); this comparison is presented in Supplementary Data 6. Results show that our prototype, which is very lightly loaded (between 0.0125 and 0.075 wt.\%) with CFs of $12 \mathrm{~mm}$ length presents a very close absorption performance, for frequencies lower than $11 \mathrm{GHz}$, to that of the commercial one, which is loaded with volatile carbon nanoparticles (carbon black). One can also note that a better performance is obtained for our prototype for high frequencies ( $\mathrm{f}>11 \mathrm{GHz}$ ).

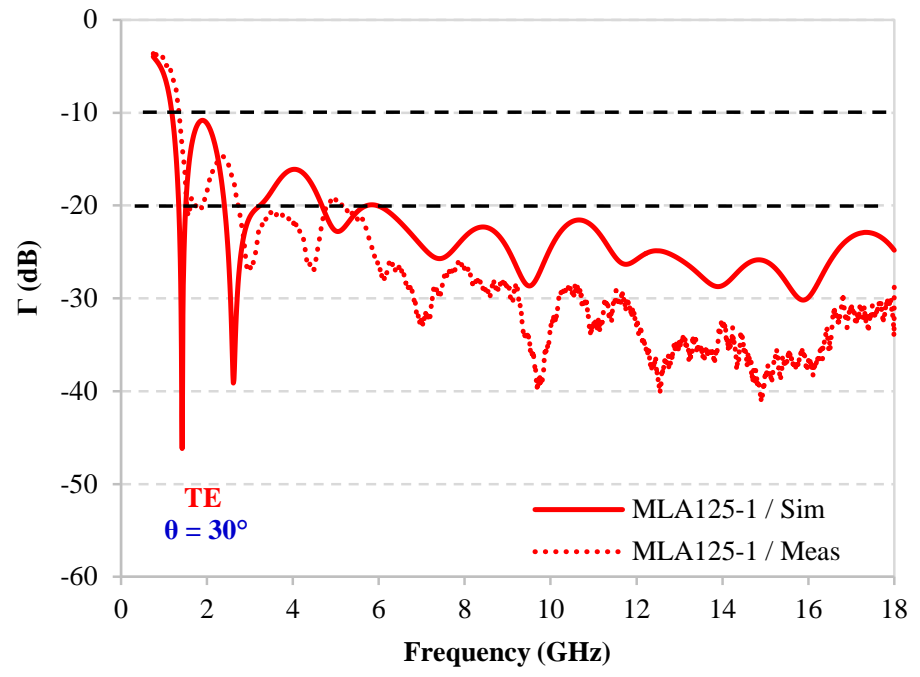

(a) 


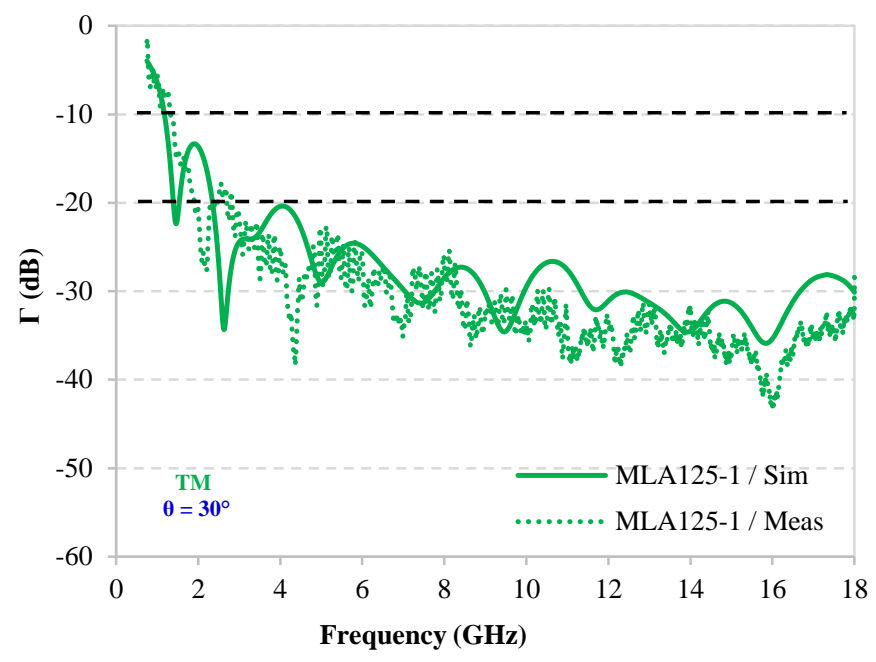

(b)

Fig. 7. Simulated and measured reflection coefficients at oblique incidence $\theta=30^{\circ}$ for (a) $\mathrm{TE}$ and

(b) TM polarizations

It can be concluded, on the one hand, that composites based on epoxy foams loaded with long carbon fibers show real potential for microwave absorption due to their low real part of permittivities associated with high losses. On the other hand, it has been demonstrated that high dielectric loss values are not necessary, or even not beneficial, for the elaboration of broadband planar absorbers, and that very slightly loaded composites allow a comparable absorption performance to that of a commercial material used in an anechoic chamber.

\subsection{Multilayer absorber design by using genetic algorithm optimizer}

In this part, the analytical method, associated with GA, is used for the optimization of the multilayer absorber. The database that was used for this optimization is composed of the same samples used before (Fig. 3). It consists of unloaded (0 wt.\%) and the loaded epoxy foam composites with $12 \mathrm{~mm} \mathrm{CF}$ length using different weight percentages between $0.0125 \mathrm{wt} . \%$ and $0.4 \mathrm{wt} . \%$; our database is therefore composed of 10 composite materials as layers' choice for the GA optimizer.

The optimization of the multilayer absorber is conducted in the frequency range between 0.75 and $18 \mathrm{GHz}$ with a targeted reflection coefficient of $-20 \mathrm{~dB}$. The used parameters for this 
optimization are listed in Table 1; the number of layers was set to 5 with a limit of thickness, between 10 and $25 \mathrm{~mm}$ for each layer, thus, the total thickness of the absorber was limited between 50 (as a minimal thickness) and $125 \mathrm{~mm}$ (as a maximal thickness).

\section{Entity Selected parameters}

Number of layers

Number of predefined materials

Minimum thickness for each layer

Maximum thickness for each layer
5

10

$10 \mathrm{~mm}$

$25 \mathrm{~mm}$

Table 1 Parameters used for the optimization of the multilayer absorber (with 5 layers) using MATLAB code with the GA

The optimized multilayer absorber MLA98-GA, proposed by the GA, presents a total thickness of $98 \mathrm{~mm}$; the chosen layers and their thicknesses are shown in Fig. 8. This design is composed first, of $10 \mathrm{~mm}$ thickness of the unloaded epoxy foam composite as a matching layer; this layer has the closest dielectric properties to that of air, so it will ensure a very good transition of the EM waves between the air and the first layer of the absorber. Then, and for the other four layers, the GA has proposed layers loaded with increasing rates of CFs: $0.0125 \mathrm{wt} . \%$, 0.025 wt. $\%, 0.075$ wt. $\%$ and 0.4 wt. $\%$ for the second, the third, the fourth and the fifth layer, respectively. The last layer is the most loaded layer among all used layers; this layer presents the highest losses and will ensure a total absorption of the EM waves. This prototype follows also the gradual impedance principle which is related to the gradual loading of the charge, as used before (MLA125-1 and MLA125-2), but with other combination of layers (compositions and thicknesses) that lead to a better absorption performance. Indeed, for the first layer, which acts as a matching layer between air and absorber, the unloaded composition ( $0 \mathrm{wt} . \%)$ is proposed, 
while the most loaded composite $(0.4$ wt.\%), with the highest dielectric losses, in order to act as an absorbing layer, is proposed for the last layer. One can note that these two layers are the same that were used for the first and last layers of the MLA125-2. For the other three layers, the gradual impedance matching, and so, gradual properties principle, is adopted with the same compositions used for the MLA125-1.

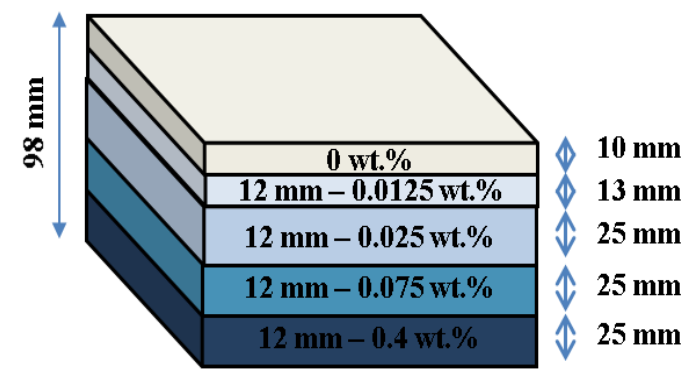

Fig. 8 MLA98-GA layers' composition and thicknesses

The different layers proposed by the GA were cut to the desired thicknesses in order to achieve the MLA98-GA prototype. The absorption performance of the latter was measured in the anechoic chamber. The final achieved MLA98-GA prototype is shown in Fig. 9 (a); the measured reflection coefficients, compared to the simulated ones, are presented in Fig. 9 (b) for normal incidence. A comparison between the calculation (by MATLAB code) and the electromagnetic simulation (CST software) of the reflection coefficients of the MLA98-GA is presented in Supplementary Data 7. The latter shows the perfect concordance between these two coefficients, thus validating the efficiency of the developed code.

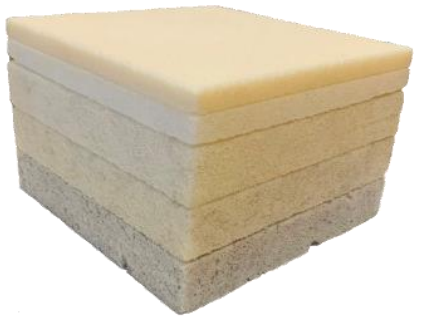

(a) 


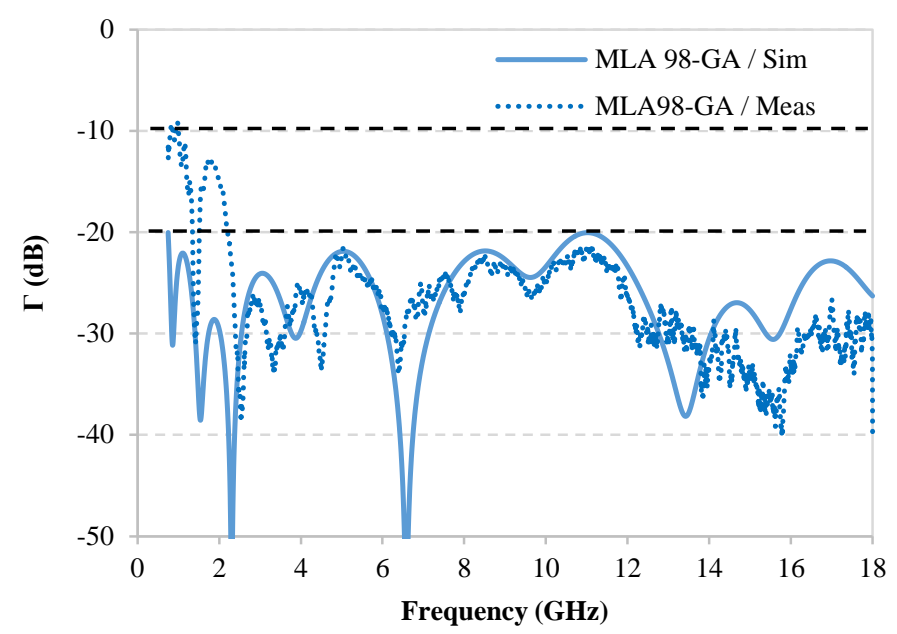

(b)

Fig. 9 (a) MLA98-GA achieved prototype and (b) comparison between the measured and simulated reflection coefficients at normal incidence

Fig. 9 (b) shows that a reflection coefficient less than $-20 \mathrm{~dB}$ is obtained by simulation in the whole studied frequency range between 0.75 and $18 \mathrm{GHz}$. This performance is confirmed by the measurement from $2.1 \mathrm{GHz}$ where a very good matching is obtained, but a degradation of the absorption performance is observed at low frequencies before $2.1 \mathrm{GHz}$ at normal and oblique incidences; this degradation comes from the limitation of the anechoic chamber used for our measurements. Indeed, even for the commercial absorber $\mathrm{AH} 125$, for which a reflection coefficient of $-20 \mathrm{~dB}$ should be obtained, as announced by SIEPEL, there is also a degradation of the performance at low frequencies below $2 \mathrm{GHz}$ (See Supplementary Data 8). In fact, below $2 \mathrm{GHz}$, we reach the limit of the anechoic chamber, and we cannot measure reflection coefficients lower than $-20 \mathrm{~dB}$.

The simulated and measured reflection coefficients for oblique incidence $\left(\theta=30^{\circ}\right)$ are presented in Fig. 10 (a) and (b) for TE and TM polarizations, respectively. By simulation, a reflection coefficient less than $-20 \mathrm{~dB}$ is obtained from $1 \mathrm{GHz}$ for TE polarization and from 0.8 $\mathrm{GHz}$ for TM polarization. This performance is confirmed by measurement from $2.4 \mathrm{GHz}$ and from $2.5 \mathrm{GHz}$ for TE and TM modes, respectively. 


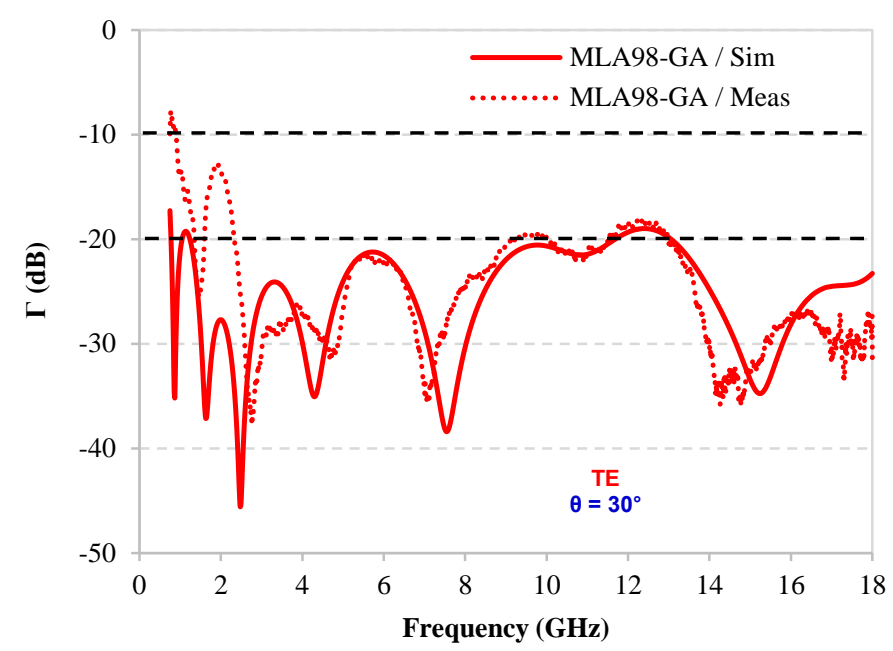

(a)

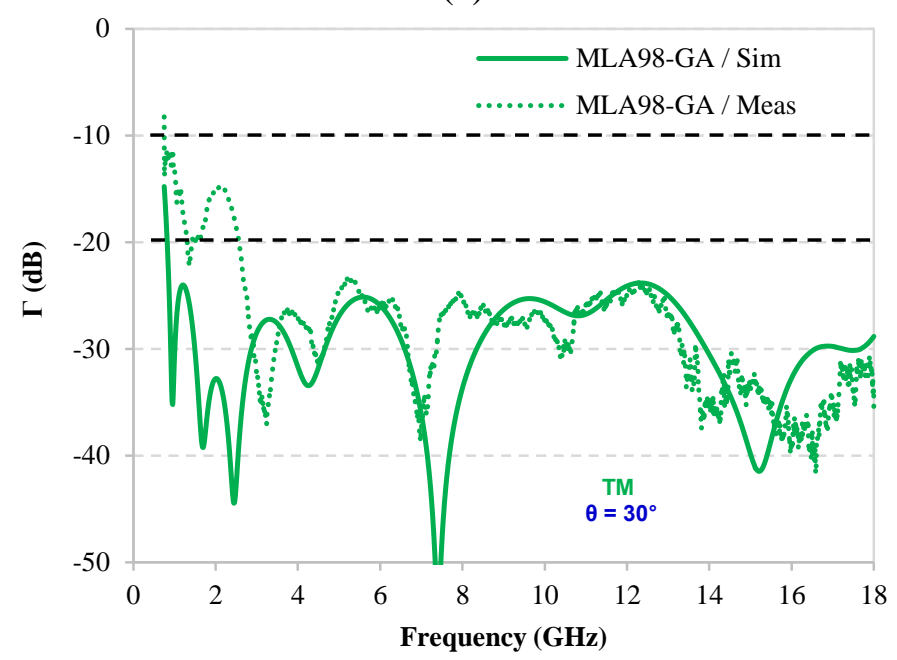

(b)

Fig. 10 Comparison between the measured and simulated reflection coefficients for MLA98-GA at oblique incidence of $30^{\circ}$ for TE (a) and TM (b) polarizations

In order to investigate the absorption mechanism of the EM waves for MLA98-GA, we have calculated the reflected power densities at each interface; results are shown in Fig. 11. For all interfaces, the reflected powers are very small $(<1.5 \%)$ beyond $2 \mathrm{GHz}$. The highest two reflected powers occur between the last two interfaces where $\operatorname{Pr} 4=1.78 \%$ and $\operatorname{Pr} 5=4.6 \%$ (@ $0.75 \mathrm{GHz}$ ) between the third and the firth and between the fourth and the fifth layers, respectively. This is due to the high difference between the load rates, and so, to the high impedance mismatch between these layers, especially between the fourth $(224 \Omega @ 0.75 \mathrm{GHz})$ and fifth layer (132 $\Omega @ 0.75 \mathrm{GHz}$ ). It should be noted here that the best absorption power of 
this prototype comes from a very good progressive absorption, and especially at low frequencies, as evidenced by the transmitted powers (Pt1 to Pt5) of Fig. 11 (b), unlike the first two prototypes (Supplementary data 5).

Otherwise, in order to confirm the relevance of the choice of the thicknesses of the first (10 $\mathrm{mm})$ and second $(13 \mathrm{~mm})$ layers, which are lower than those used for commercial absorbers (25 $\mathrm{mm}$ ), a simulation of MLA125-3 prototype (Supplementary Data 9 (a)), based on the same layer compositions as those of MLA98-GA, was conducted with the same thickness of $25 \mathrm{~mm}$ for each layer (total thickness of $125 \mathrm{~mm}$ ). A comparison of the simulated reflection coefficients of the MLA98-GA and MLA125-3, for normal and oblique incidence $\left(\theta=30^{\circ}\right)$ of the EM waves, is presented in Supplementary Data 9. The latter shows that, for the normal incidence (Supplementary Data 9 (b)), a better performance is obtained by MLA98-GA, especially at low ([1.2-7.2] GHz) and high ([12.2-16.2] GHz) frequencies, associated with a slight degradation, in comparison with the MLA125-3, for frequencies between [7.2-12.3] GHz and for frequencies higher than $16 \mathrm{GHz}$. One can note that even in these frequency ranges, the MLA98-GA presents a reflection coefficient lower than $-26 \mathrm{~dB}$, which is equivalent to $99.7 \%$ of absorption of the incident EM waves. The same conclusions can be done for the simulated reflection coefficients for oblique incidence of $30^{\circ}$ (Supplementary Data 9 (c) and (d)). Finally, this comparison shows that the choice of the composition and the thickness of each layer is both very important for optimizing the absorption performance of a multilayer absorber, and thus confirms, moreover, the importance of using the GA optimizer. 


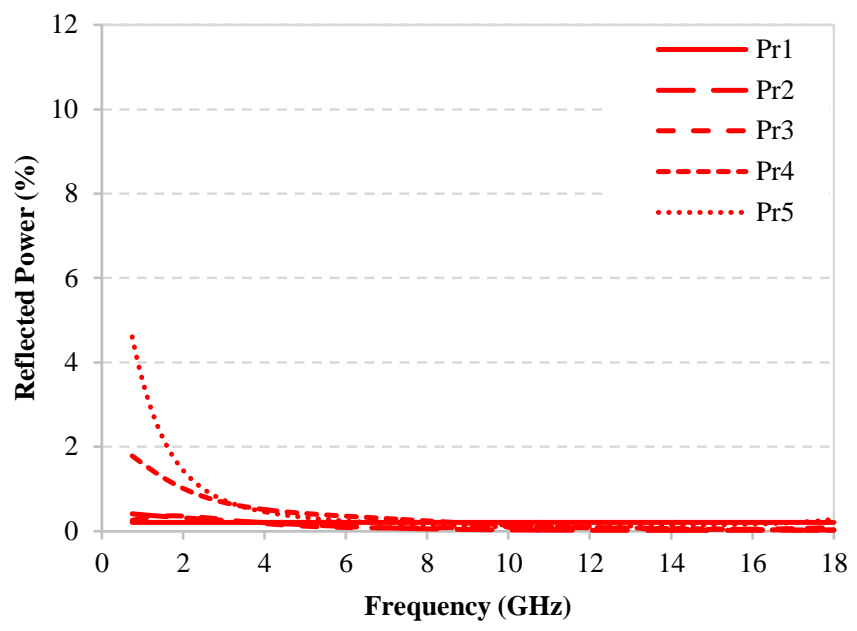

(a)

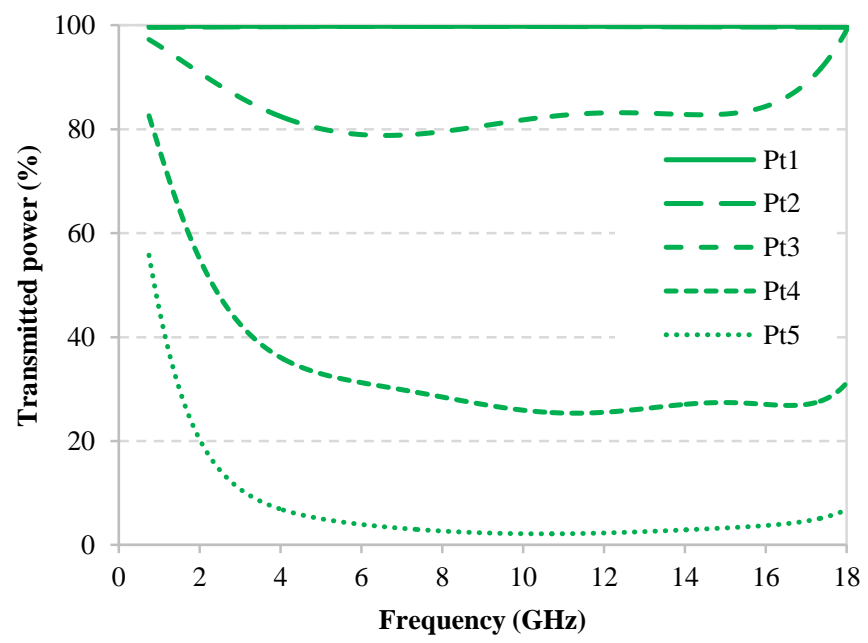

(b)

Fig. 11 (a) Reflected $\left(\operatorname{Pr}_{N}\right)$ and (b) transmitted $\left(\mathrm{Pt}_{\mathrm{N}}\right)$ power densities for MLA98-GA

\subsection{Comparison of the absorption performance of the prototypes}

Fig. 12 shows the comparison between the measured reflection coefficients, at normal incidence, of the MLA125-1, the MLA98-GA and the commercial absorber AH 125; this Figure shows close reflection coefficients. From the one hand, MLA125-1 and AH 125 present nearly the same performance between 1.8 and $10.7 \mathrm{GHz}$ while MLA125-1 has a better absorption performance beyond $10.7 \mathrm{GHz}$. From the other hand, MLA98-GA has a better absorption performance than that of $\mathrm{AH} 125$ between 0.75 and $5 \mathrm{GHz}$ and between $12 \mathrm{GHz}$ and $18 \mathrm{GHz}$, and this is while having a reduction of $22 \%$ in the total thickness (from $125 \mathrm{~mm}$ to $98 \mathrm{~mm}$ ). However, between $8 \mathrm{GHz}$ and $12 \mathrm{GHz}, \mathrm{AH} 125$ presents a better absorption performance than 
that of MLA98-GA, but this latter presents a reflection coefficient less than $-21.5 \mathrm{~dB}$ in this frequency range, which corresponds to absorption higher than 99.3.\%.

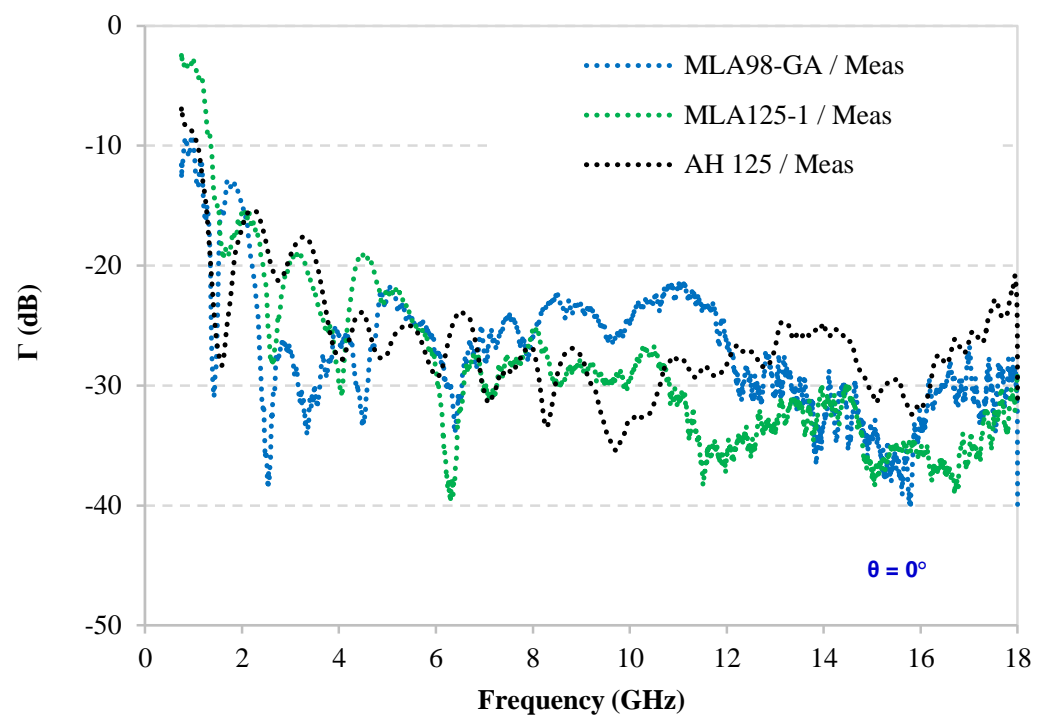

Fig. 12 Comparison between the measured reflection coefficients of MLA98-GA, MLA1251and AH 125 (commercial absorber) at normal incidence

In order to better compare the performances of the obtained prototypes, which present different thicknesses, a factor of merit (FoM) that takes into account the total thickness of the multilayer absorber (Thickness) and the total measured reflection coefficient $\left(\Gamma_{\text {linear }}\right)$, is introduced. The FoM is calculated using Eq. (13) and it is inversely proportional to the Thickness and $\Gamma_{\text {linear }}$, which are the parameters to reduce.

$$
F O M=\frac{1}{\text { Thickness } * \Gamma_{\text {linear }}}
$$

By comparing the calculated FoM of the different achieved prototypes with that of the commercial absorber AH 125 (Fig. 13), we observe that MLA125-1 prototype presents a better FoM at high frequencies than that of the commercial absorber. Likewise, MLA98-GA presents a better FoM at low $(<7 \mathrm{GHz})$ and high $(>12 \mathrm{GHz})$ frequencies than that of $\mathrm{AH} 125$ with a thickness reduction of $22 \%$. 


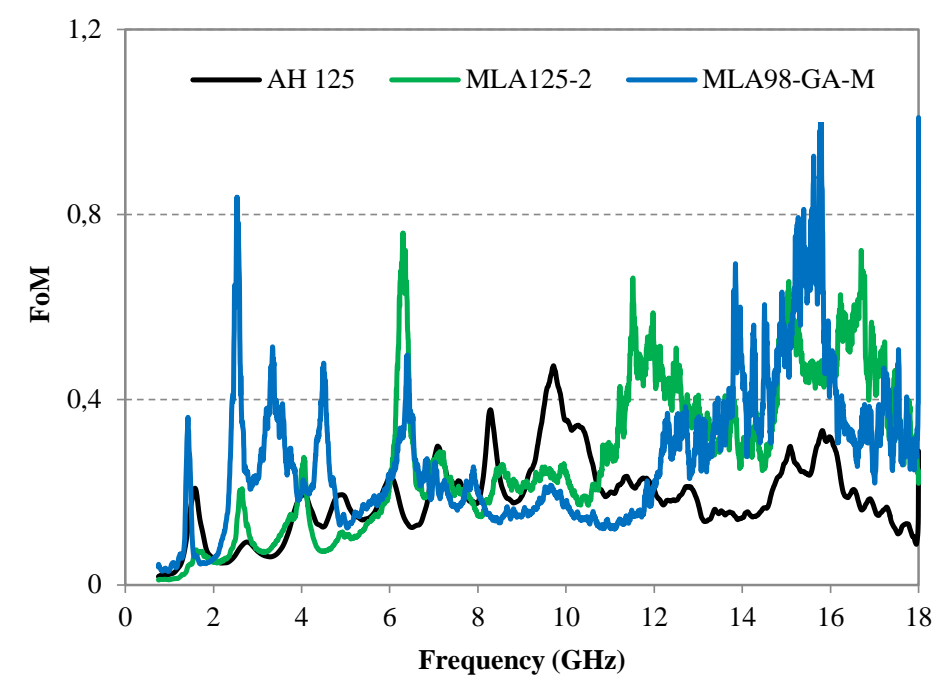

Fig. 13 Calculated factor of merit of MLA125-1and MLA98-GA compared to that of AH 125

In conclusion, the MLA98-GA prototype, optimized by the GA, shows a very good absorption performance in the entire studied frequency band between 0.75 and $18 \mathrm{GHz}$. It also shows a better performance than the commercial absorber AH 125 and with a lower thickness. Moreover, both MLA98-GA and MLA125-1 prototypes present better absorption/thickness compromises than that of the commercial absorber over a large part of the studied frequency band. Thus, these results show, on the one hand, the potential of the composites based on epoxy foam loaded with very low rates of long fibers for the realization of performant electromagnetic absorbers, and on the other hand, the importance of the use of an optimizer, such as GA, to improve the absorption performance while reducing the thickness of the planar multilayer absorber.

\section{Conclusion}

In this work, composites based on epoxy foam loaded with different weight percentages (between 0 wt.\% and 0.4 wt.\%) of carbon fibers of $12 \mathrm{~mm}$ length are used to design planar multilayer absorbers. Two strategies were used for the design of these broadband absorbers. The first one is based on the gradual impedance principle and therefore, on the choice of successive gradually loaded layers (with gradual dielectric properties). Thus, two prototypes were proposed; for the first one, very slightly loaded composites (CF rates between 0.0125 wt.\% and 
0.075 wt.\% with a step of 0.0125 ) are used and for the second prototype, relatively more loaded composites (CF rates between 0 wt.\% and 0.4 wt.\% with a step of 0.1 ) are employed. For both prototypes, the number of layers was set to five and the thickness of each layer was fixed to 25 $\mathrm{mm}$; thus, the total thickness of the multilayer absorbers is $125 \mathrm{~mm}$. Results show that a better absorption performance is obtained, in all the studied frequency range [0.75-18] GHz, for the slightly loaded prototype (MLA125-1) with a reflection coefficient less than $-10 \mathrm{~dB}$ from 1.3 $\mathrm{GHz}$ and less than $-20 \mathrm{~dB}$ from $4.1 \mathrm{GHz}$. These results also show that the choice of the composition of the different layers is important and that the high dielectric properties (permittivities and losses) are not advantageous for planar absorbers because they induce high reflections at the different interfaces. We obtain a very good concordance between simulated and measured reflection coefficients. Close absorption bandwidth and performance to that of the commercial absorber (AH 125), in the entire studied frequency range, were obtained with our prototype. These obtained results confirm that CF loaded epoxy foam materials with very low $\mathrm{CF}$ rates (from $0.0125 \mathrm{wt} . \%$ to $0.075 \mathrm{wt} . \%$ ) are suitable to achieve a planar multilayer absorber.

The second used strategy to design a planar multilayer absorber is to optimize the choice of the composition and the thickness of each layer by using the genetic algorithm optimizer. A database of ten materials is used and a minimum and maximum thickness of $10 \mathrm{~mm}$ and $25 \mathrm{~mm}$, respectively, for each layer, are imposed to the optimizer program. MLA98-GA prototype is thereby proposed with gradual load of compositions (0 wt.\%, 0.0125 wt.\%, 0.025 wt.\%, 0.075 wt. $\%$ and 0.4 wt. $\%$ ) for the successive layers in depth, and with a total thickness of $98 \mathrm{~mm}$ of the absorber. Here, a reduction of the total thickness of $22 \%$ is obtained, compared to that of the commercial absorber AH 125. The measurement of the realized MLA98-GA prototype shows a very good concordance between the simulated and the measured reflection coefficients, confirming by this, the best absorption performance that was observed by a comparison of all the achieved prototypes and also with the commercial absorber AH 125. In addition to that, the factor of merit that qualifies the performance of the prototypes, taking into consideration their 
total thickness and their reflection coefficients, was calculated. It shows a better factor for our prototypes than that of the commercial absorber $\mathrm{AH} \mathrm{125,} \mathrm{which} \mathrm{means} \mathrm{that} \mathrm{our} \mathrm{realized}$ prototypes present a better compromise between absorption and thickness.

Finally, the MLA-98 optimized by GA shows the best result (absorption and factor of merit) which confirms the interest of using GA for the optimization of a planar absorber, because it allows obtaining the best combination of composition/thickness for each layer of this absorber.

\section{Acknowledgments}

This work was supported by the European Union through the European Regional Development Fund and by the Ministry of Higher Education and Research, Brittany Région, Côtes d'Armor Département and Saint Brieuc Armor Agglomération through the CPER Projects MATECOM and SOPHIE-STICC. Authors want to thank J. Sol for his support for the prototypes measurement.

\section{Conflict of Interest Statement}

The authors declare that they have no conflict of interest

\section{References}

1. Sista KS, Dwarapudi S, Kumar D, et al (2021) Carbonyl iron powders as absorption material for microwave interference shielding: A review. Journal of Alloys and Compounds 853:157251. https://doi.org/10.1016/j.jallcom.2020.157251

2. Micheli D, Apollo C, Pastore R, et al (2011) Nanostructured composite materials for electromagnetic interference shielding applications. Acta Astronautica 69:747-757. https://doi.org/10.1016/j.actaastro.2011.06.004 
3. Mishra M, Singh AP, Gupta V, et al (2016) Tunable EMI shielding effectiveness using new exotic carbon: Polymer composites. Journal of Alloys and Compounds 688:399-403. https://doi.org/10.1016/j.jallcom.2016.07.190

4. Industries that benefit of Comtest Engineering EMC Solutions. In: Comtest Engineering. https://www.comtest.us/industries/. Accessed 3 May 2021

5. Klemperer CJ von, Maharaj D (2009) Composite electromagnetic interference shielding materials for aerospace applications. Composite Structures 91:467-472. https://doi.org/10.1016/j.compstruct.2009.04.013

6. Bowen Bai, Xiaoping Li, Jin Xu, Yanming Liu (2015) Reflections of Electromagnetic Waves Obliquely Incident on a Multilayer Stealth Structure With Plasma and Radar Absorbing Material. IEEE Transactions on Plasma Science 43:2588-2597. https://doi.org/10.1109/TPS.2015.2447536

7. Raghavan S, Rajeshkumar V (2013) An Overview of Metamaterials in Biomedical Applications. 4

8. Medical Industry related Solutions. In: Comtest Engineering. https://www.comtest.us/industries/medical/. Accessed 3 May 2021

9. Weijia Duan, Chen Han, Sun Mingming, et al A composite electromagnetic absorber for anechoic chambers. In 2013 Proceedings of the International Symposium on Antennas \& Propagation, pp. 412-414.

10. Microwave Absorbers. In: Siepel. https://siepel.asia/products/rf-microwave/microwaveabsorbers/. Accessed 2 Nov 2020 
11. Méjean C, Pometcu L, Benzerga R, et al (2017) Electromagnetic absorber composite made of carbon fibers loaded epoxy foam for anechoic chamber application. Materials Science and Engineering: B 220:59-65. https://doi.org/10.1016/j.mseb.2017.03.009

12. Polystyrene Absorbers | Eco-friendly. In: Comtest Engineering. https://www.comtest.us/components/polystyrene-absorbers/. Accessed 3 May 2021

13. Holloway CL, DeLyser RR, German RF, et al (1997) Comparison of electromagnetic absorber used in anechoic and semi-anechoic chambers for emissions and immunity testing of digital devices. IEEE Transactions on Electromagnetic Compatibility 39:33-47. https://doi.org/10.1109/15.554693

14. Chen M, Zhu Y, Pan Y, et al (2011) Gradient multilayer structural design of CNTs/SiO2 composites for improving microwave absorbing properties. Materials \& Design 32:30133016. https://doi.org/10.1016/j.matdes.2010.12.043

15. Thomassin J-M, Jérôme C, Pardoen T, et al (2013) Polymer/carbon based composites as electromagnetic interference (EMI) shielding materials. Materials Science and Engineering: R: Reports 74:211-232. https://doi.org/10.1016/j.mser.2013.06.001

16. Lederer, P.G. (1986). An introduction to radar absorbent materials (Report No. 85016). Royal Signals and Radar Establishment, Malvern, England

17. Méjean C (2017) Élaboration de Nouveaux Matériaux absorbants: Application en Chambres anéchoïques. PhD, University of Rennes 1

18. Li Y, Shen B, Yi D, et al (2017) The influence of gradient and sandwich configurations on the electromagnetic interference shielding performance of multilayered thermoplastic polyurethane/graphene composite foams. Composites Science and Technology 138:209216. https://doi.org/10.1016/j.compscitech.2016.12.002 
19. Dang S, Lin Y, Wei X, Ye H (2018) Design and preparation of an ultrawideband gradient triple-layered planar microwave absorber using flaky carbonyl iron as absorbent. Journal of Materials Science: Materials in Electronics 29:17651-17660. https://doi.org/10.1007/s10854-018-9869-3

20. Bizhani H, Nayyeri V, Khanjarian M, Ramahi OM (2020) Gradient composite microwave absorber: Investigation into loading profiles of conductive nanofiller. Journal of Applied Physics 127:014902. https://doi.org/10.1063/1.5122970

21. Zhang XF, Zhou GP (2015) Broadband asymmetric electromagnetic wave absorption tailored by impedance gradation: To construct diode-like absorbers. Materials Letters 149:29-32. https://doi.org/10.1016/j.matlet.2015.02.099

22. Ji B, Fan S, Kou S, et al (2021) Microwave absorption properties of multilayer impedance gradient absorber consisting of Ti3C2TX MXene/polymer films. Carbon 181:130-142. https://doi.org/10.1016/j.carbon.2021.05.018

23. De Lustrac A (2018) Matériaux composites en électromagnétisme - Matériaux absorbants radar, Électronique - Photonique | Technologies radars et applications. Techniques de l'Ingénieur, 10 Sept. 2018.

24. Micheli D, Apollo C, Pastore R, Marchetti M (2010) X-Band microwave characterization of carbon-based nanocomposite material, absorption capability comparison and RAS design simulation. Composites Science and Technology 70:400-409. https://doi.org/10.1016/j.compscitech.2009.11.015

25. Goldberg DE, Kuo CH (1987) Genetic Algorithms in Pipeline Optimization. Journal of Computing in Civil Engineering 1:128-141. https://doi.org/10.1061/(ASCE)08873801(1987)1:2(128) 
26. Zhang Q, Barri K, Jiao P, et al (2021) Genetic programming in civil engineering: advent, applications and future trends. Artificial Intelligence Review 54:1863-1885. https://doi.org/10.1007/s10462-020-09894-7

27. Riechmann T (2001) Genetic algorithm learning and evolutionary games. Journal of Economic Dynamics and Control 25:1019-1037. https://doi.org/10.1016/S01651889(00)00066-X

28. Ding Y, Shi Y, Wang A, et al (2020) Block-oriented correlation power analysis with bitwise linear leakage: An artificial intelligence approach based on genetic algorithms. $\begin{array}{llll}\text { Future } & \text { Generation } & \text { Computer } & \text { Systems }\end{array}$ https://doi.org/10.1016/j.future.2019.12.046

29. Ye F, Qi W, Xiao J (2011) Research of Niching Genetic Algorithms for Optimization in Electromagnetics. $\quad$ Procedia 16:383-389. https://doi.org/10.1016/j.proeng.2011.08.1099

30. Reddaf A, Djerfaf F, Ferroudji K, et al (2020) Modeling of electromagnetic behavior of composite thin layers using genetic algorithm. Mathematics and Computers in Simulation 167:281-295. https://doi.org/10.1016/j.matcom.2018.12.011

31. Kent S, Günel T (1997) Dielectric Permittivity Estimation of Cylindrical Objects Using Genetic Algorithm. Journal of Microwave Power and Electromagnetic Energy 32:109-113. https://doi.org/10.1080/08327823.1997.11688331

32. Ibnyaich S, Wakrim L, Hassani MM (2021) Nonuniform Semi-patches for Designing an Ultra Wideband PIFA Antenna by Using Genetic Algorithm Optimization. Wireless Personal Communications 117:957-969. https://doi.org/10.1007/s11277-020-07905-y 
33. Soontornpipit P, Furse CM, You Chung Chung (2005) Miniaturized biocompatible microstrip antenna using genetic algorithm. IEEE Transactions on Antennas and Propagation 53:1939-1945. https://doi.org/10.1109/TAP.2005.848461

34. Suraj P, Behera BR, Badhai RK (2020) Optimization of Metamaterials-Based Wi-Fi Antenna Using Genetic Algorithm. National Academy Science Letters 43:333-337. https://doi.org/10.1007/s40009-020-00876-5

35. Weile DS, Michielssen E (2001) The control of adaptive antenna arrays with genetic algorithms using dominance and diploidy. IEEE Transactions on Antennas and Propagation 49:1424-1433. https://doi.org/10.1109/8.954931

36. Goswami B, Mandal D (2013) A genetic algorithm for the level control of nulls and side lobes in linear antenna arrays. Journal of King Saud University - Computer and Information Sciences 25:117-126. https://doi.org/10.1016/j.jksuci.2012.06.001

37. Wang L, Liu H-T (2021) Parameter optimization of bidirectional re-entrant auxetic honeycomb metamaterial based on genetic algorithm. Composite Structures 267:113915. https://doi.org/10.1016/j.compstruct.2021.113915

38. Zhang J, Wang G, Wang T, Li F (2021) Genetic Algorithms to Automate the Design of Metasurfaces for Absorption Bandwidth Broadening. ACS Applied Materials \& Interfaces 13:7792-7800. https://doi.org/10.1021/acsami.0c21984

39. Duan B, Zhang J, Wang P, et al (2020) Design and preparation of an ultrathin broadband metamaterial absorber with a magnetic substrate based on genetic algorithm. Journal of $\begin{array}{llll}\text { Magnetism } & \text { and } & \text { Magnetic }\end{array}$ https://doi.org/10.1016/j.jmmm.2020.166439 
40. Singh S, Sinha A, Zunke RH, et al (2018) Double layer microwave absorber based on $\mathrm{Cu}$ dispersed SiC composites. Advanced Powder Technology 29:2019-2026. https://doi.org/10.1016/j.apt.2018.05.008

41. Padhy S, De A, Debata RR, Meena RS (2018) Design, Characterization, and Optimization of a Multilayer U-type Hexaferrite-Based Broadband Microwave Absorber. IEEE Transactions on Electromagnetic Compatibility 60:1734-1742. https://doi.org/10.1109/TEMC.2018.2805364

42. Kumar A, Singh S, Singh D (2017) Development of Double Layer Microwave Absorber Using Genetic Algorithm. IOP Conference Series: Materials Science and Engineering 234:012009. https://doi.org/10.1088/1757-899X/234/1/012009

43. Kurniawan AF, Anwar MS, Nadiyyah K, et al (2021) Thickness optimization of a doublelayered microwave absorber combining magnetic and dielectric particles. Materials Research Express 8:065001. https://doi.org/10.1088/2053-1591/ac04ea

44. Breiss H, El Assal A, Benzerga R, et al (2020) Long Carbon Fibers for Microwave Absorption: Effect of Fiber Length on Absorption Frequency Band. Micromachines 11:1081. https://doi.org/10.3390/mi11121081

45. Pometcu L (2016) Matériaux et forme innovants pour l'atténuation en Hyper Fréquences. $\mathrm{PhD}$, University of Rennes 1

46. Fenner RA, Rothwell EJ, Frasch LL (2012) A comprehensive analysis of free-space and guided-wave techniques for extracting the permeability and permittivity of materials using reflection-only measurements: EXTRACTION OF MATERIAL PROPERTIES. Radio Science 47:n/a-n/a. https://doi.org/10.1029/2011RS004755 
47. Duan Y, Guan H (2017) Microwave absorbing materials. Pan Stanford Publishing, Singapore

48. Qin F, Brosseau C (2012) A review and analysis of microwave absorption in polymer composites filled with carbonaceous particles. Journal of Applied Physics 111:061301. https://doi.org/10.1063/1.3688435

49. Balanis CA (1989) Advanced engineering electromagnetics. Wiley, New York

50. Genetic Algorithm Options - MATLAB \& Simulink - MathWorks France. https://fr.mathworks.com/help/gads/genetic-algorithm-options.html. Accessed 14 Jun 2021 
Design and optimization of ultra-wideband planar multilayer absorber based

\title{
on long-carbon fibers-loaded composites
}

\author{
Aicha El Assal $^{1}$, Hanadi Breiss ${ }^{1}$, Ratiba Benzerga ${ }^{1, *}$, Ala Sharaiha ${ }^{1}$ \\ ${ }^{1}$ CNRS, IETR - UMR 6164, Univ Rennes 1, F-35000 Rennes, France \\ *Corresponding Author: ratiba.benzerga@univ-rennes1.fr
}

\section{Supplementary Data 1.}

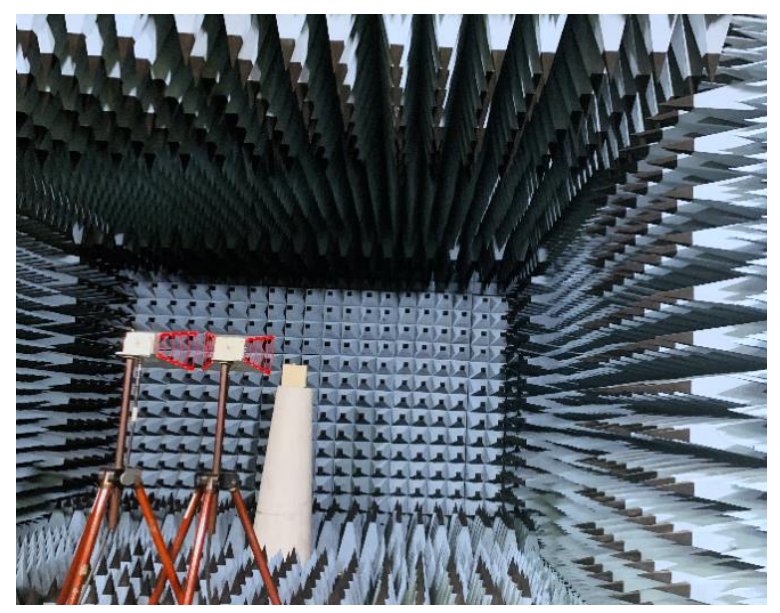

(a)

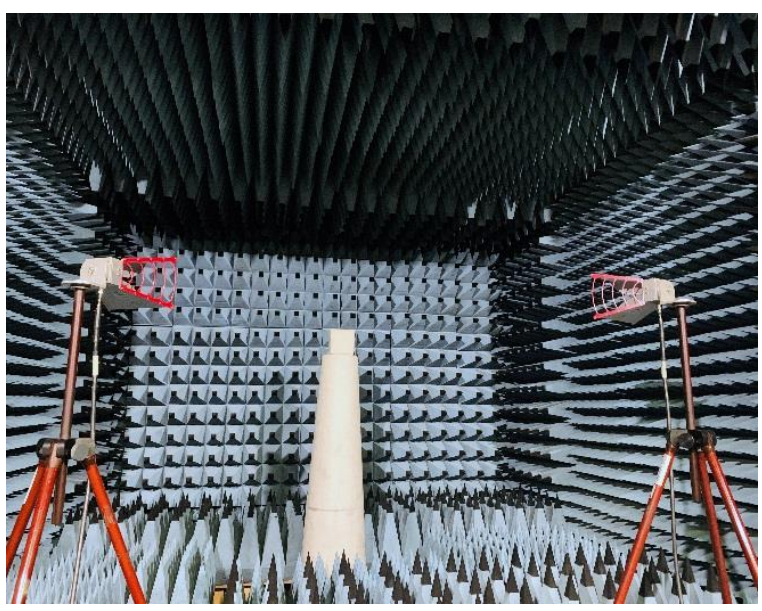

(b)

Supplementary Data 1. Configuration of the measurement in the anechoic chamber for (a) normal incidence and (b) oblique incidence of $30^{\circ}$. 


\section{Supplementary Data 2.}

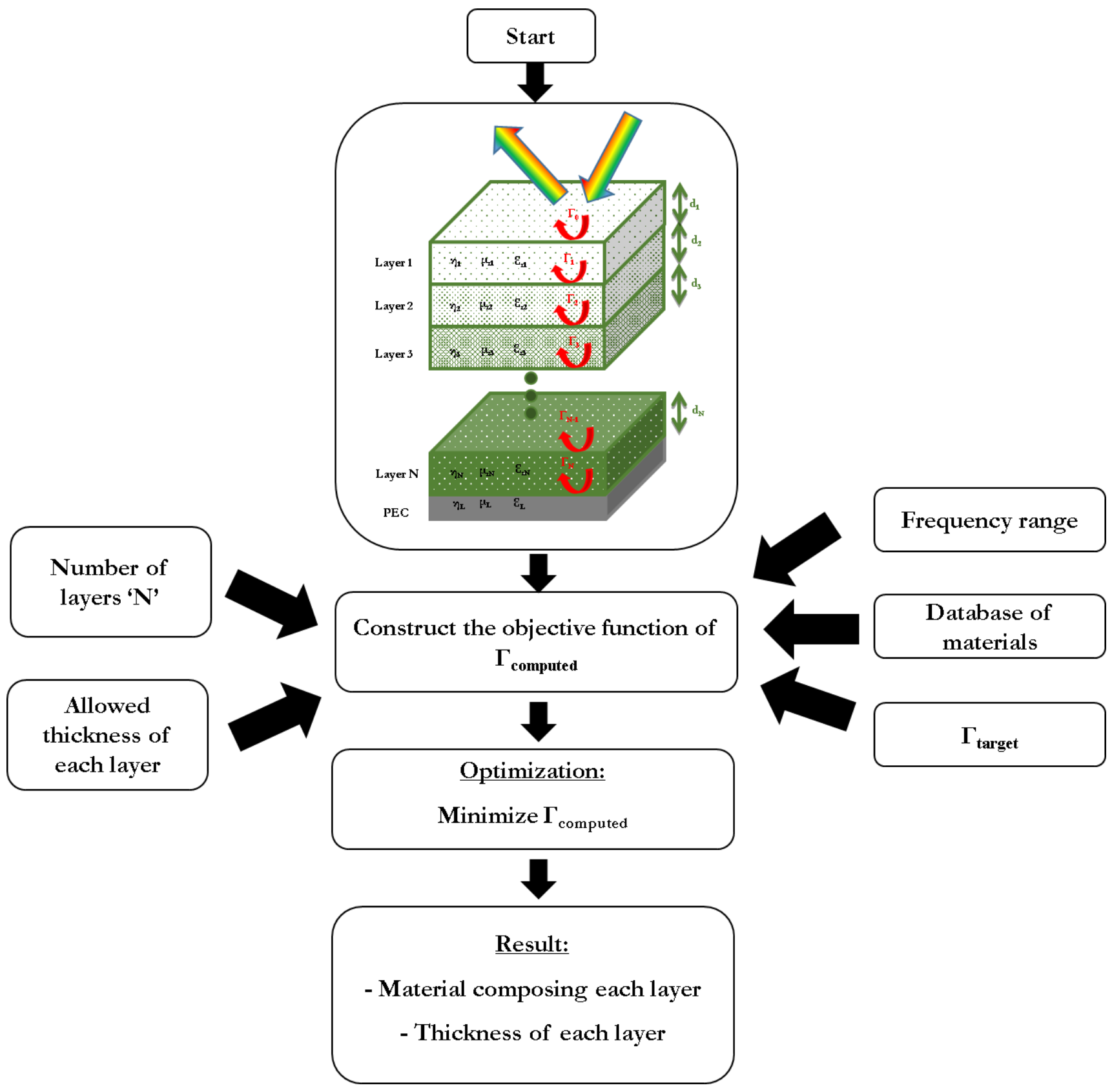

Supplementary Data 2. Flowchart showing the procedure for the design of multilayer absorber using the $G A$ optimizer in $M A T L A B$. 


\section{Supplementary Data 3.}

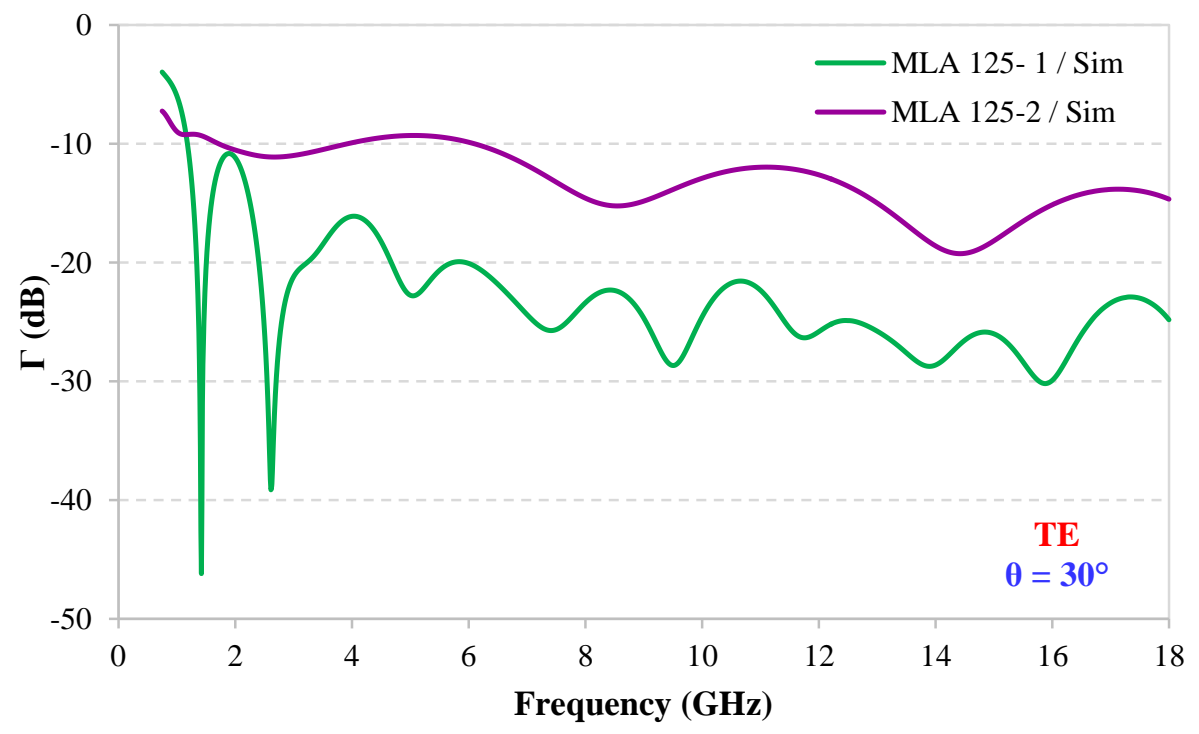

Supplementary Data 3. Comparison between the simulated reflection coefficients of MLA125-1 and MLA125-2 for TE polarization of the oblique incidence of $30^{\circ}$. 


\section{Supplementary Data 4.}

The characteristic impedances $\eta_{c}$ of composite materials are calculated using equation (1) where $\mu_{\mathrm{r}}$ and $\varepsilon_{\mathrm{r}}^{\prime}$ are respectively the complex permeability and complex permittivity of the composite [1], [2]

$$
\eta_{c}=\eta_{0} \sqrt{\frac{\mu_{r}}{\varepsilon_{r}}}=\sqrt{\frac{\mu_{0}}{\varepsilon_{0}}} \sqrt{\frac{\mu_{r}}{\varepsilon_{r}}}=\sqrt{\frac{\mu_{0}}{\varepsilon_{0}}} \sqrt{\frac{1}{\varepsilon_{r}}}
$$

[1] F. Qin and C. Brosseau, "A review and analysis of microwave absorption in polymer composites filled with carbonaceous particles," Journal of Applied Physics, vol. 111, no. 6, p. 061301, Mar. 2012, doi: 10.1063/1.3688435.

[2] Y. Duan and H. Guan, Microwave absorbing materials. 2017.

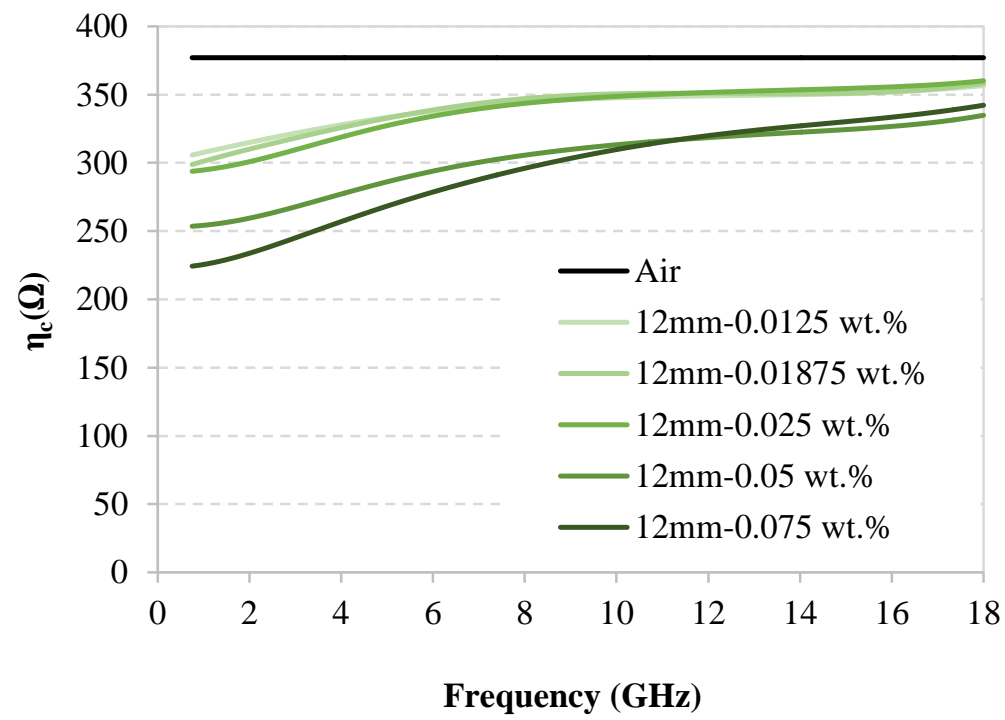

(a)

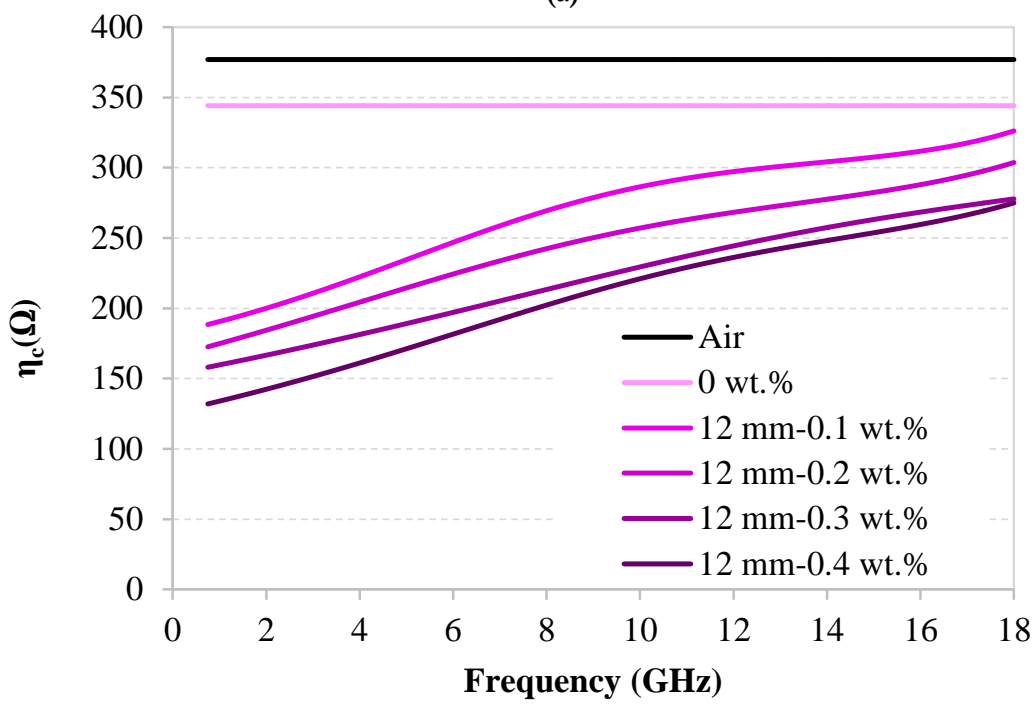

(b)

Supplementary Data 4. Calculated module of characteristic impedances of the different composites used for (a) MLA125-1 and (b) MLA125-2. 


\section{Supplementary Data 5.}

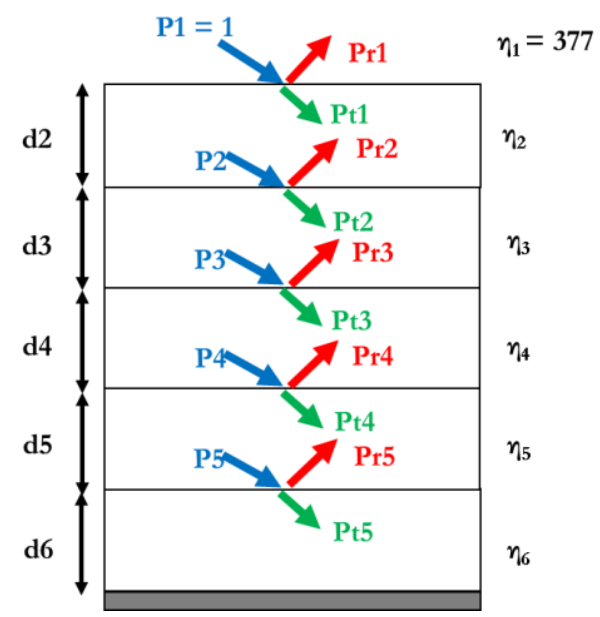

(a)

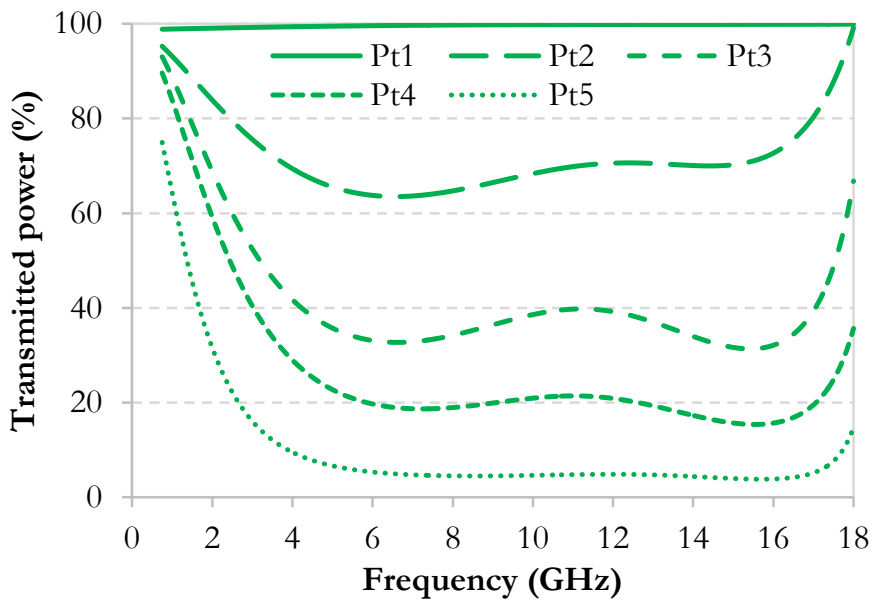

(b)

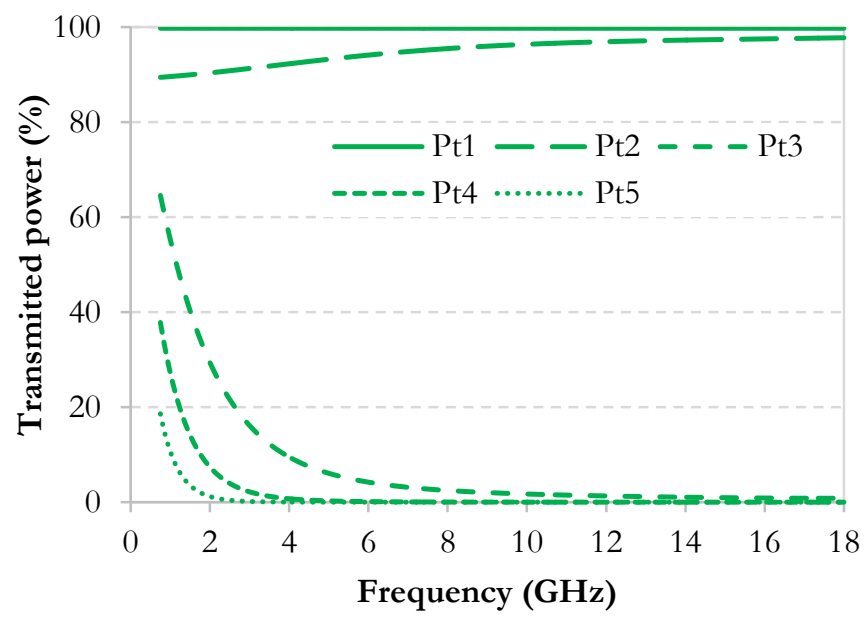

(c)

Supplementary Data 5. (a) Reflection and transmission power densities of the EM waves for a multilayer absorber and the calculated transmitted power densities $P t_{N}$ for (b) MLA125-1 and (c) MLA125-2. 


\section{Supplementary Data 6.}

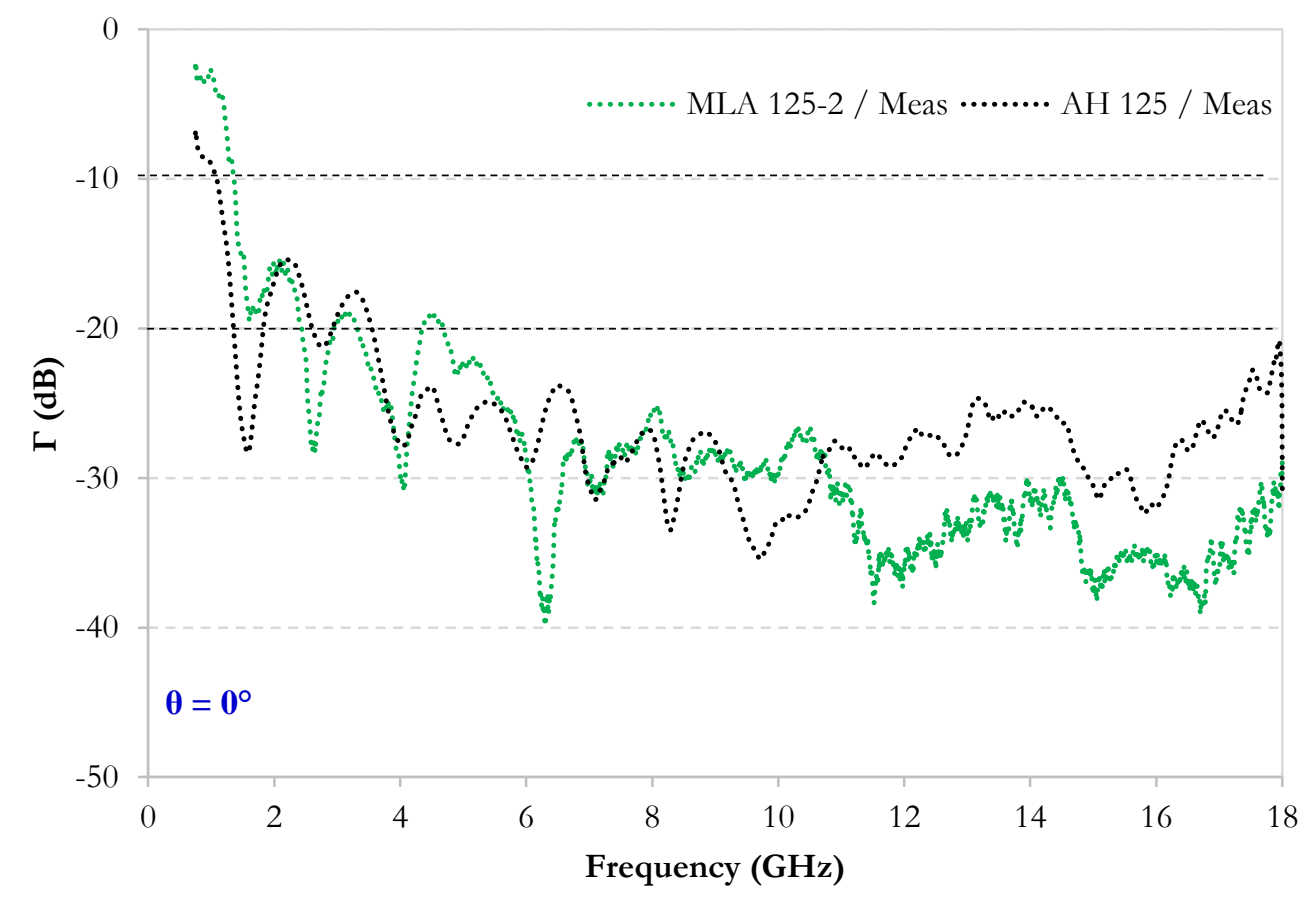

Supplementary Data 6. Comparison between the measured reflection coefficients of MLA125-1 and AH 125. 


\section{Supplementary Data 7.}

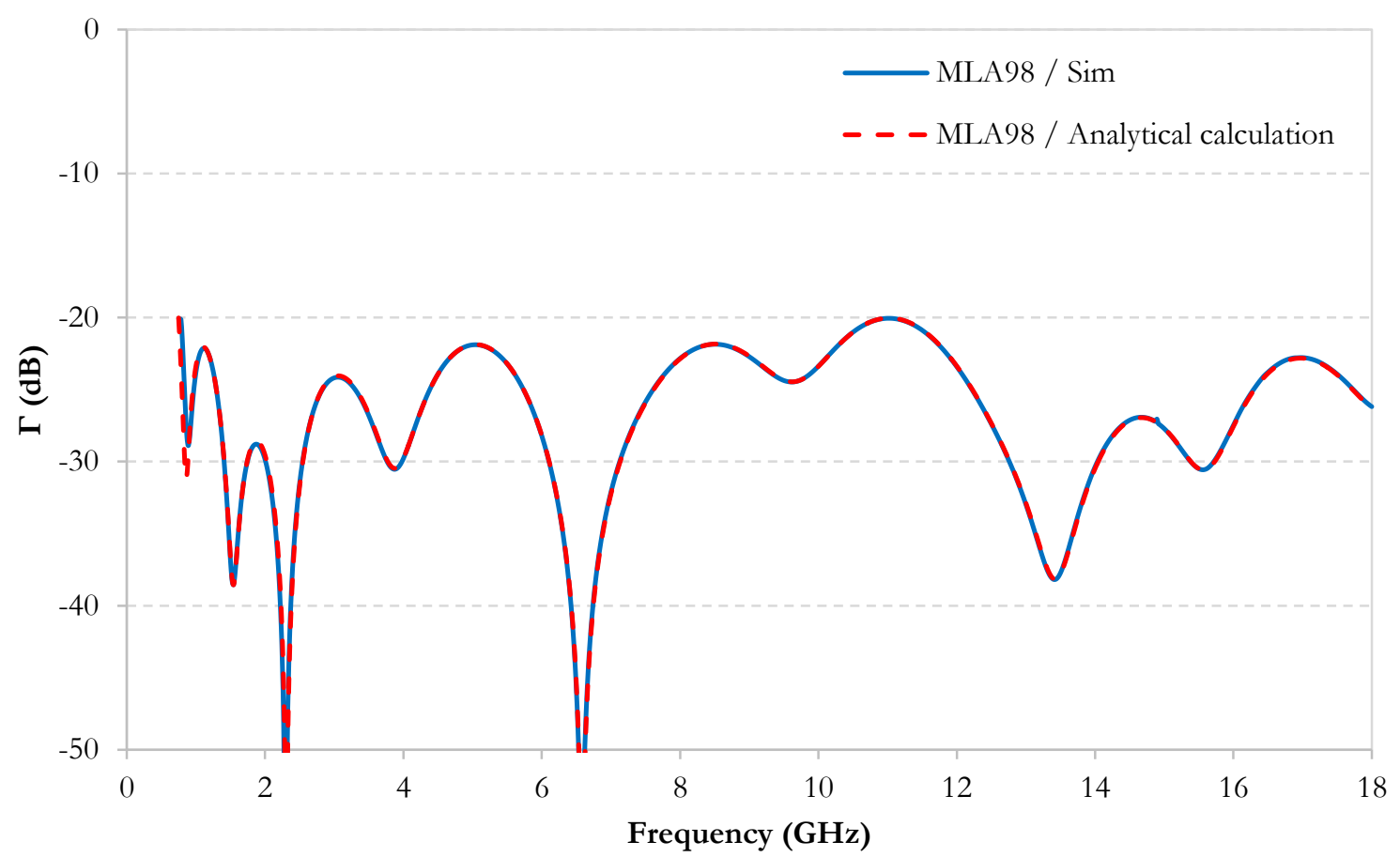

Supplementary Data 7. A comparison between the calculated (by MATLAB code) and the simulated (CST software) reflection coefficients of the MLA98-GA 


\section{Supplementary Data 8.}

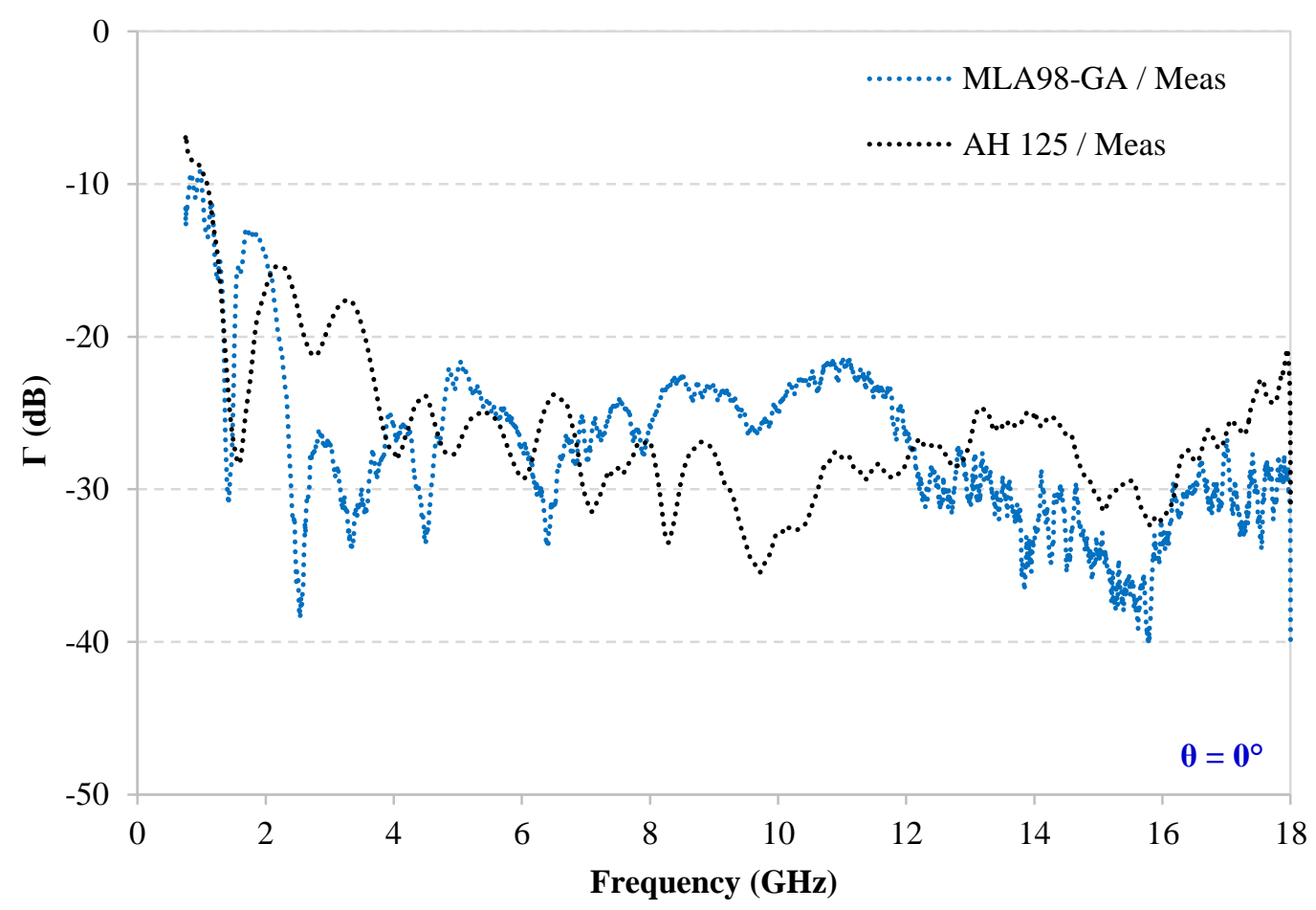

Supplementary Data 8. Comparison between the measured reflection coefficients of MLA98-GA and AH 125. 


\section{Supplementary Data 9.}

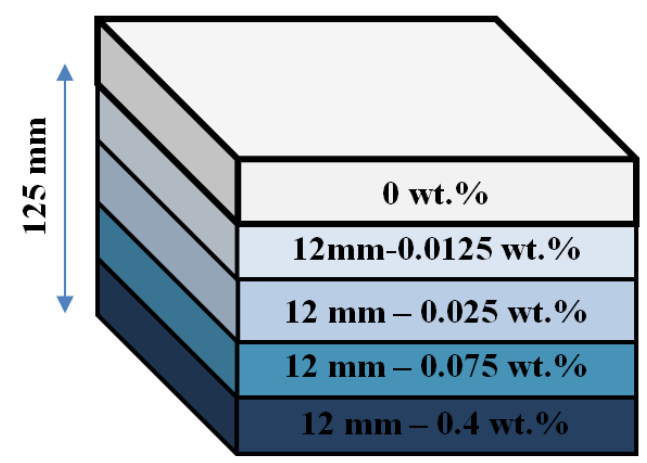

(a)

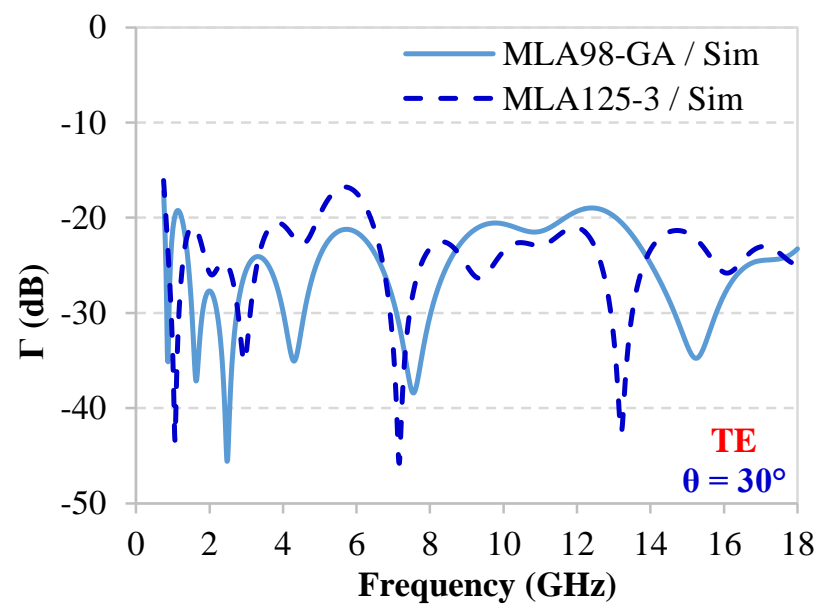

(c)

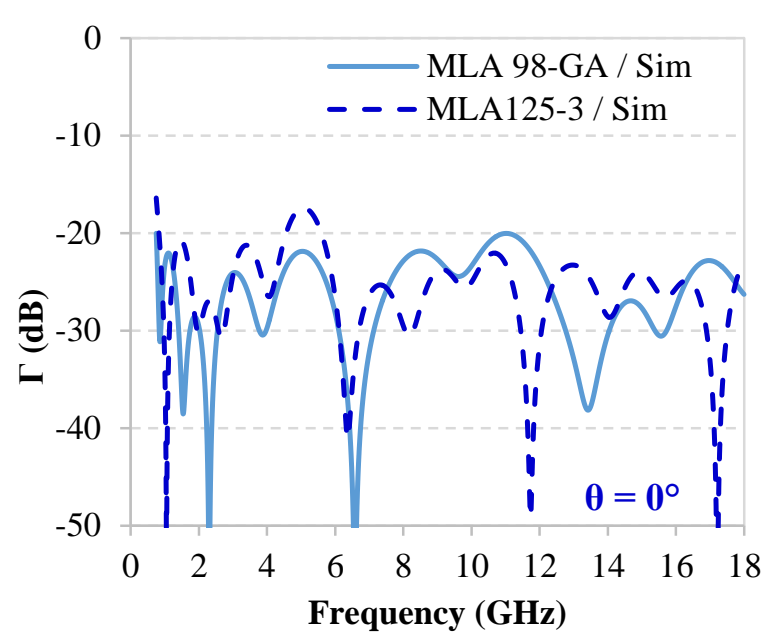

(b)

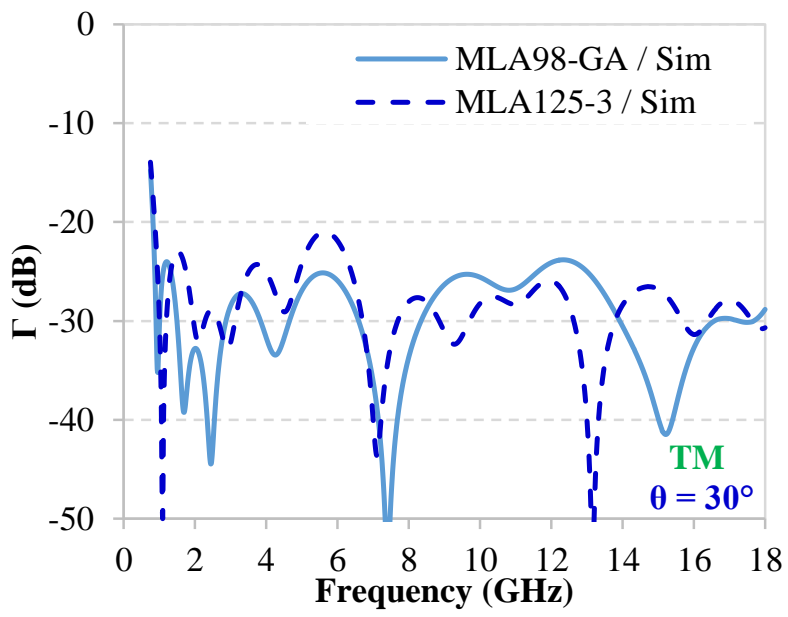

(d)

Supplementary Data 9. (a) MLA125-3 and comparison between the simulated reflection coefficients of MLA98$G A$ and MLA125-3 at (b) normal incidence and at oblique incidence of $30^{\circ}$ for (c) TE and (d) TM polarizations. 